\title{
Energy dependence of the differential photoelectron cross sections of molecular nitrogen ${ }^{\text {a) }}$
}

\author{
Donald M. Mintz ${ }^{\text {b) }}$ and Aron Kuppermann

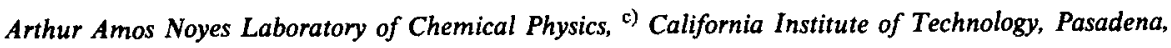 \\ California 91125 \\ (Received 14 March 1978)
}

\begin{abstract}
The angular distribution of photoelectron intensity for molecular nitrogen was studied using $\mathrm{He} I$ and $\mathrm{Ne} I$ resonance line discharge light sources. Studies of photcelectron angular distributions covering a range of photon energies, and thus a range of photoelectron energies, are possible using the weaker high order lines in each discharge as well as the principal lines. Peaks in three photoelectron bands of $\mathrm{N}_{2}$ were studied at the photon energies $16.85,19.78,21.22,23.09$, and $23.74 \mathrm{eV}$, where possible. We find that the $v^{\prime}=0$ peak of the $X^{2} \Sigma_{g}^{+}$band has abnormally high intensity and, at the higher photon energies, an abnormally low angular distribution asymmetry parameter, $\beta$. Several mechanisms for this anomaly are discussed, including autoionization, the variation of electric dipole transition moments with internuclear distance, and possible shape resonance phenomena. None of these explanations is completely in agreement with all theoretical and experimental evidence.
\end{abstract}

\section{INTRODUCTION}

One of the fundamental assumptions used extensively in the analysis of molecular photoelectron spectra is that vibrational peak intensities of a given photoionization band are proportional to the corresponding FranckCondon nuclear wavefunction overlap factors between ground and ionic states. ${ }^{1}$ While this assumption is not exact, it is usually sufficiently accurate to be of considerable use in the interpretation of those spectra. ${ }^{2}$

Recent measurements of the Her photoelectron spec$\mathrm{tra}^{3}$ and angular distributions $\mathrm{s}^{4-6}$ for vibrational peaks of the ground $X^{2} \Sigma_{g}^{+}$state of the $\mathrm{N}_{2}^{+}$appear to violate this assumption. We have reinvestigated the photoelectron angular distributions of $\mathrm{N}_{2}$ at several photon wavelengths in the hopes of resolving the controversy which has arisen.

\section{THEORETICAL BACKGROUND}

The differential photoelectron cross section $d \sigma / d \Omega$ for direct photoionization from an initial state $\Psi_{n}(r, R)$ to a final state $\Psi_{m}(r, R)$ relates to the square of the absolute value of the corresponding transition matrix element by ${ }^{7}$ :

$$
\frac{d \sigma}{d \Omega}=\frac{(2 \pi)^{2} m k_{f} \omega}{\hbar^{2} c}\left|\left\langle\Psi_{m}(\mathbf{r}, \mathbf{R})|\mathbf{P} \cdot e \mathbf{r}| \Psi_{n}(\mathbf{r}, \mathbf{R})\right\rangle\right|^{2}
$$

in the dipole length representation. Here $\mathbf{r}$ and $\mathbf{R}$ denote the set of electronic and nuclear coordinates, respectively, $\mathbf{P} \cdot \mathbf{r}$ is the operator for the interaction between the molecular electrons and the electromagnetic field where $P$ is the light polarization vector, and $\Omega$ denotes the angles which specify the direction of photoemission in a coordinate system determined by the direction of polarization of the light and the molecular orientation. Also, $k_{f}$ and $\omega$ are, respectively, the wavenumber of

\footnotetext{
a) Work supported in part by the Department of Energy (Contract No. EY-76-S-03-767). Report Code: CALT-767P4-157.

b) Work performed in partial fulfillment of the requirements for the $\mathrm{Ph} . \mathrm{D}$. degree in Chemistry at the California Institute of Technology. Present address: TRW Defense and Space Systems Group, Redondo Beach, CA 90278.

${ }^{c)}$ Contribution No. 5746 .
}

the ejected photoelectron and the photon frequency. Further, $m$ and $e$ a re the electron mass and charge, and $c$ is the speed of light. In the Born-Oppenheimer approximation, ${ }^{8}$ each total wavefunction is factored into the product of a nuclear wavefunction $\chi(R)$ and an electronic wavefunction $\psi(\mathbf{r} ; \mathbf{R})$. For sufficiently high temperatures and if rotational structure is not resolved, it has been shown that the average over rotational sublevels becomes an average over initial molecular orientations. ${ }^{9}$ Finally, for ionization by nonpolarized light, we average $d \sigma / d \Omega$ over orientations of the polarization vector with respect to the photon propagation vector. The result is the familiar form ${ }^{10}$ :

$$
I_{\substack{u \rightarrow f \\ \rightarrow \rightarrow v^{\prime}}}(\theta)=\frac{Q_{i \rightarrow f}^{v \rightarrow v^{\prime}}}{4 \pi}\left[1-\frac{\beta}{4}\left(3 \cos ^{2} \theta-1\right)\right]
$$

where $\theta$ is the angle between the direction of electron emission and the photon propagation vector, and $Q_{i \rightarrow f}^{v \rightarrow v^{\prime}}$ is the total rotationally averaged cross section for transition from the vibrational level $v$ of the initial neutral electronic state $i$ to level $v^{\prime}$ of the final electronic state $f$ of the ion. For such direct photoionization, if the electronic transition moment is independent of final vibrational state, the asymmetry parameter $\beta$ is a function of the electronic levels involved in the transition and the energy of the outgoing electron (and thus the incident photon wavelength); it does not, however, depend on the final vibrational state. FranckCondon factors are incorporated in the total cross section $Q_{i \rightarrow f}^{v-v^{\prime}}$. The value of $\beta$ may range ${ }^{11}$ from -1 to +2 .

Theoretical calculations of the asymmetry parameter $\beta$ have been made for an extensive collection of atoms and molecules ${ }^{12-17}$ using approximations of different degrees of validity. In all cases, for photoelectron energies $\gtrsim 200 \mathrm{eV}, \beta$ varies slowly with that energy, but close to ionization threshold, that variation is more pronounced. However, rates of variations of $\beta$ with energy higher than $+0.4 \mathrm{eV}^{-1}$ are uncommon. These normally accompany only autoionization of Rydberg states above the first ionization potential, as has been observed in xenon. ${ }^{18}$ Here, the measured $\beta$ values take the form ${ }^{17}$ : 


$$
\beta=\frac{Q^{\mathrm{dir}} \beta_{\mathrm{dir}}+Q^{\text {auto }} \beta_{\text {auto }}}{Q^{\text {dir }}+Q^{\text {auto }}},
$$

where the indices dir and auto refer to direct and autoionization pathways, respectively.

Thus, even for studies of photoelectron angular distributions measured near threshold, over a limited range of photoelectron energies, we expect a gradual variation of $\beta$ for ionization of electrons from a single molecular orbital. For ionization by light of a fixed wavelength, ions with increasing degrees of vibrational excitation correspond to decreasing amounts of translational energy of the outgoing electrons. To a first approximation and in the absence of autoionization, the variation of $\beta$ across the vibrational envelope of an electronic band should only reflect the variation of $\beta$ with electron energy. Deviations of the measured values of $\beta$ for certain vibrational levels within a given electron band from a monotonic curve involving the rest of those levels and corresponding gross deviations of measured relative vibrational intensities from the calculated Franck-Condon factors should thus indicate the presence of molecular autoionization, other ionization pathways, or a breakdown of the assumptions leading to the proportionality of vibrational intensities to those factors.

\section{EXPERIMENTAL}

The variable angle photoelectron spectrometer used in these experiments was described in detail elsewhere,$^{19}$ and will be described here very briefly. It consists essentially of a rare gas resonance discharge lamp, a rotatable hemispherical electron energy analyzer of $6.800 \mathrm{~cm}$ mean radius, electrostatic lenses, and a channel electron multiplier. The analyzer is attached to a sample chamber which is normally filled with 4 mTor $r$ of the substance being studied $\left(\mathrm{N}_{2}\right.$ in the present case), as measured by a high pressure ionization gauge.

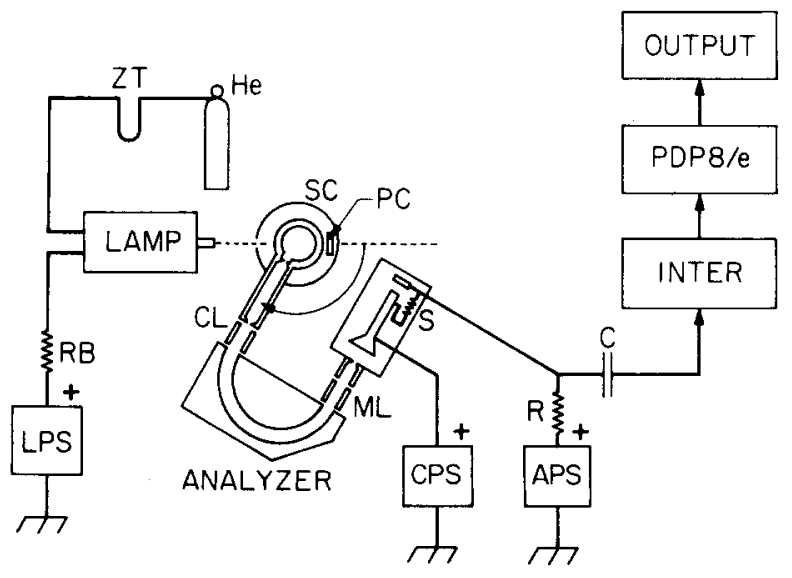

FIG. 1. Block diagram of variable angle photoelectron spectrometer. He, cylinder of UHP helium; $\mathrm{ZT}$, liquid nitrogen immersed zeolite trap for lamp helium supply; RB, lamp ballast resistor, $1320 \Omega$; LPS, lamp power supply, $555 \mathrm{~V}, 300 \mathrm{~mA}$ maximum; SC, sample chamber; PC, photocathode for light flux measurement; $C L$, electron lens elements before hemispherical analyzer; ANALYZER, $180^{\circ}$ hemispherical electrostatic electron energy analyzer; ML, electron lens elements between hemispheres and detector.
TABLE I. Table of atomic emission lines used.

\begin{tabular}{lllc}
\hline \hline Light source & $\begin{array}{l}\text { Wavelength } \\
(\AA)\end{array}$ & $\begin{array}{l}\text { Energy } \\
(\mathrm{eV})\end{array}$ & Intensity \\
\hline He I & 584.334 & 21.217 & 100 \\
& 537.030 & 23.086 & 2 \\
& 522.213 & 23.741 & 0.5 \\
Ne I & 735.895 & 16.847 & 100 \\
& 626.822 & 19.779 & 0.3 \\
\hline \hline
\end{tabular}

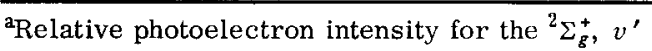
$=0, \mathrm{~N}_{2}^{+}$line for each lamp gas.

Spectra of photoelectron intensity versus electron energy are generated using a minicomputer-based data acquisition system. A block diagram of the apparatus is given in Fig. 1.

We investigated the photoelectron angular distributions for nitrogen at the wavelengths listed in Table $\mathrm{I}_{0}{ }^{20}$ Normally, photoelectron spectra taken with the unfiltered radiation of a windowless discharge must be interpreted with caution. A weak feature could be caused by an ionization with low transition probability, by one of the Her or Ner higher order lines, or by impurity lines in the light source. Impurity lines from neutral atom emissions, at $\mathrm{Hr}, \mathrm{NI}$, or $\mathrm{Cr}$, have energies substantially lower than those of the principal lamp emission lines. Thus, corresponding photoelectron energies are much lower than those of the $584 \AA$ spectra and lie outside of the energy range scanned in the present investigation. The ionic states populated by $584 \AA$ light have been well characterized; we expect no structure due to inforseen new ionic states in the photon range 16.85-23.74 eV covered by this experiment. Further, population of high-lying ionic levels, such as the $C^{2} \Sigma_{u}^{+}$state, ${ }^{21-23}$ should produce photoelectrons at low kinetic energies, again, out of the range of the present investigation.

The higher order lines present in the discharge lamp have low intensity and give rise to photoelectron spectra shifted with respect to those of the principal emission by a known amount and superimposed upon that of the principal emission. For fixed angle studies, these higher order lines are in general an undesired feature, but we have found them of considerable use in studying the variation of $\beta$ values with electron energy. Except for $v^{\prime} \geq 3$ of the $A^{2} \Pi_{u}$ band and the entire $B^{2} \Sigma_{u}^{+}$band of $\mathrm{N}_{2}$, weak structure resulting from ionization by the higher order lines does not overlap the structure produced by the intense principal atomic lines. The photoelectron spectrum of $\mathrm{N}_{2}$ has sharp structure of high intensity and our apparatus has relatively high sensitivity. Thus, the photoelectron intensity produced by the higher order lines and amounting to less than $2 \%$ of the intensity of their more intense counterparts was adequate for this study. This required long data acquisition times, as long as $10 \mathrm{~h}$ for the study of a single peak, and angular distributions over many of the weaker peaks were reproduced as many as 5 times. Fortunately, the photoelectrons arising from the higher order lines fall in a region of high electron energy where background 


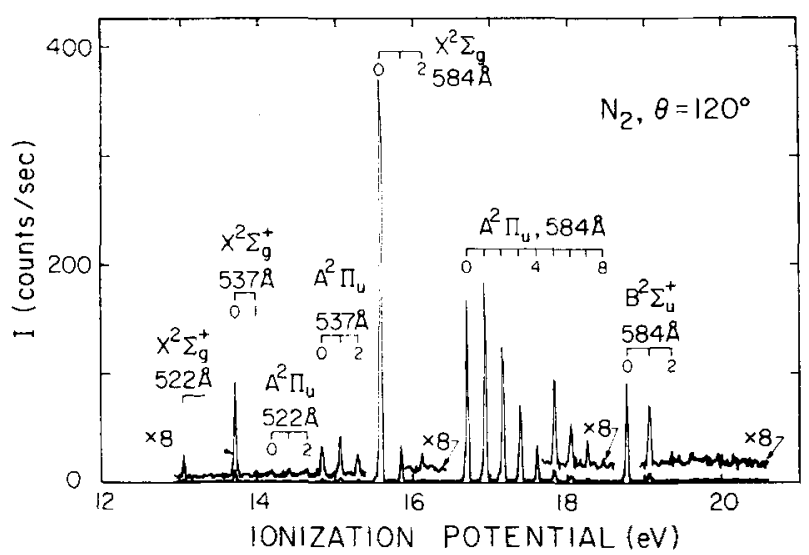

FIG. 2, He I photoelectron spectrum of $\mathrm{N}_{2}$ at $\theta=120^{\circ}$. The spectrum was accumulated over 511 data channels, an incremented energy of $15 \mathrm{meV}$ between successive channels, and a dwell time per channel of $30 \mathrm{sec}$. Background was not subtracted from the spectrum displayed here.

rates are low, under 1 count per sec. We find no photoelectron peaks corresponding to weak atomic lines other than those listed by Kelley. ${ }^{20}$

Photoelectron intensities measured as peak heights by the PDP8/e data acquisition software at each of nine detector angles in the range $\theta=40^{\circ}-120^{\circ}$ were corrected for background contributions and for the volume of intersection of the lamp and detector view cones at each $\theta$ and were compensated for the linear variation of counting rate with sample pressure. These intensities were then fitted to $\mathrm{Eq}$. (2) by a weighted least squares procedure. The quoted error limits in the resulting values of $\beta$ reflect both goodness of fit to Eq. (2) and reproducibility.

Correction for photoelectron background is a crucial part of making accurate measurements of $\beta$, especially when the signal to background ratio becomes as low as $1: 2$, as it does in a few of our experiments. During operation, we parameterize an electron-energy-dependent

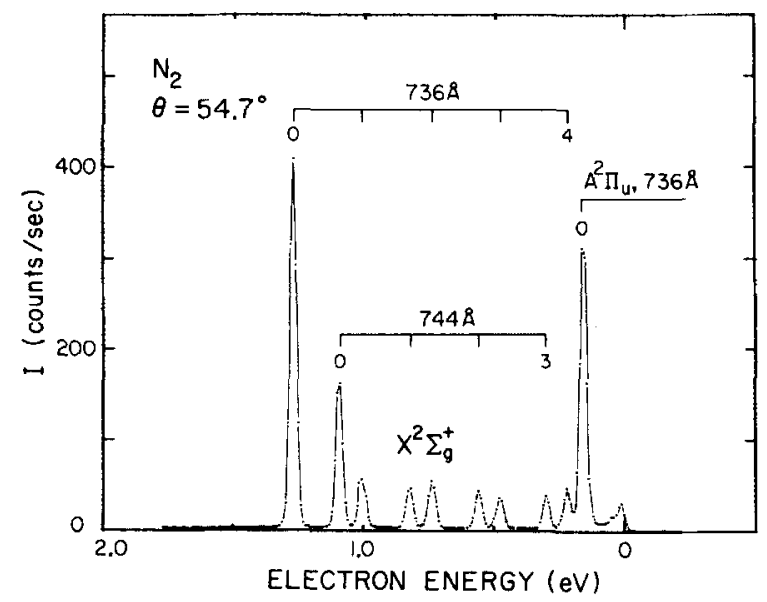

FIG. 3. Ne I photoelectron spectrum of $\mathrm{N}_{2}$ at $\theta=54.7^{\circ}$, including only the 736 and $744 \AA$ spectra. The spectrum was accumulated with $10 \mathrm{meV}$ incremented between channels, and with a dwell time of $45 \mathrm{sec}$. Background was not subtracted from the spectrum displayed here.

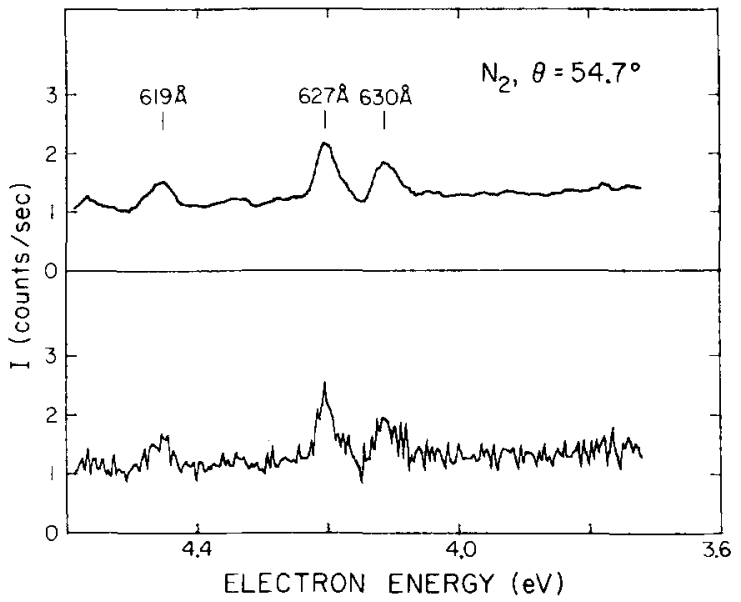

FIG. 4. Ne I spectrum of $\mathrm{N}_{2}$ at $\theta=54.7^{\circ}$, showing $v^{\prime}=0 X^{2} \Sigma_{g}^{+}$ peaks produced by the 619,627 , and $630 \AA$ lines in the discharge. Spectrum was accumulated over 218 channels, an incremented energy of $4 \mathrm{meV}$ per channel, and at a dwell time of $90 \mathrm{sec}$. The lower trace is the raw spectrum and the upper trace is a 13-point smoothing of the lower trace.

background as a series of three connected straight line segments. The typical background spectrum increases roughly at a rate of $1 / 6$ count $\mathrm{eV}^{-1} \mathrm{sec}^{-1}$ over the range of decreasing electron energy from $13 \mathrm{eV}$ (background near zero) to $1 \mathrm{eV}$.

The PDP8/e data reduction computer program subtracts the parameterized background measured for each of the nine angles of an angular distribution before calculating $B$ values. In the case of the $627 \AA$ angular distributions of the $v^{\prime}=0 X^{2} \Sigma_{g}^{+}$peak, background counting rates were measured at the corresponding electron energy immediately after measuring the angular distribution, without the abovementioned parameterization.

This background subtraction procedure seems to yield accurate results for fairly weak peak intensities. For example, in studies ${ }^{24}$ we have made of the ionization of the $2 p$ subshell of neon, this procedure yields good agreement with previous theoretical and experimental results for signal to background ratios in the range 1.6-13. 3 .

\section{RESULTS}

Spectra taken with the Her and Ner lamps arepresented in Figs. 2 and 3, respectively. Photoelectron peaks corresponding to $v^{\prime}=0$ of the $X^{2} \Sigma_{g}^{+}$ion produced by the higher order lines of the neon discharge are shown separately in Fig. 4. Of the several lines in those spectra, we measured $\beta$ only for the $627 \AA$ one. Because of intensity reasons, we made no attempts to measure $\beta$ values for $v^{\prime}=1$ of the $X^{2} \Sigma_{g}^{*}$ state at 627 or $522 \AA$. Signal to background ratios were no better than $1: 1$ for $v^{\prime}=0$ and we expected the $v^{\prime}=1$ peaks to be weaker by an order of magnitude. The values of $\beta$ obtained in these these studies are listed in Table II.

\section{A. $X^{2} \Sigma_{g}^{+}$band}

In agreement with previous measurements by Morgenstern et al. ${ }^{5}$ Carlson, ${ }^{4}$ and Hancock and Samson, ${ }^{8} \beta$ 
TABLE II. Asymmetry parameter $\beta$ for ionization of $\mathrm{N}_{2}$ by photons of different wavelength.

\begin{tabular}{|c|c|c|c|c|c|c|c|c|c|}
\hline \multirow{2}{*}{$\begin{array}{l}\text { Ionic } \\
\text { state }\end{array}$} & \multirow[b]{2}{*}{$v^{\prime}$} & \multirow{2}{*}{$\begin{array}{l}\text { I. P. } \\
\text { (ev) }\end{array}$} & \multicolumn{2}{|l|}{$584 \AA$} & \multicolumn{2}{|l|}{$736 \mathrm{~A}$} & \multirow[b]{2}{*}{$537 \AA$} & \multirow[b]{2}{*}{$522 \AA$} & \multirow[b]{2}{*}{$627 \AA$} \\
\hline & & & Experimental & Theory & Experimental & Theory & & & \\
\hline \multirow[t]{4}{*}{$X^{2} \Sigma_{g}^{+}$} & 0 & 15.58 & $\begin{array}{l}0.74 \pm 0.02 \\
\left(0.5,{ }^{\mathrm{a}} 0.69,{ }^{\mathrm{b}} 0.68,{ }^{\mathrm{c}} 0.9^{\mathrm{d}}\right)\end{array}$ & $\begin{array}{l}1.91,^{\mathrm{e}} 0.68^{\mathrm{f}} \\
1.01^{\mathrm{e}}\end{array}$ & $\begin{array}{c}1.00 \pm 0.03 \\
\left(1.20,^{2} 0.99^{b}\right)\end{array}$ & $\begin{array}{l}1.93,^{e} 0.31^{1} \\
0.99^{8}\end{array}$ & $0.72 \pm 0.03$ & $0.95 \pm 0.05$ & $\overline{1.3 \pm 0.2}$ \\
\hline & 1 & 15.85 & $\begin{array}{l}1.43 \pm 0.02 \\
\left(1.4,{ }^{\mathrm{a}} 1.40,{ }^{\mathrm{b}} 1.5^{\mathrm{c}}\right)\end{array}$ & & $\begin{array}{l}0.93 \pm 0.04 \\
\left(0.92^{b}\right)\end{array}$ & & $1.1 \pm 0.2$ & & \\
\hline & 2 & 16.12 & $\begin{array}{l}1.3 \pm 0.1 \\
\left(0.73^{b}\right)\end{array}$ & & $0.22 \pm 0.04$ & & & & \\
\hline & 3 & 16.39 & & & $0.14 \pm 0.04$ & & & & \\
\hline \multirow[t]{7}{*}{$A^{2} \Pi_{u}$} & 0 & 16.69 & $\begin{array}{l}0.36 \pm 0.02 \\
\left(0.43,{ }^{b} 0.37,{ }^{c} 0.9^{\mathrm{d}, \mathrm{h}}\right)\end{array}$ & 0.72 & $-0.26 \pm 0.11$ & & $0.7 \pm 0.1$ & & \\
\hline & 1 & 16.92 & $\begin{array}{l}0.29+0.02 \\
\left(0.30,,^{2, \mathrm{~h}} 0.37^{\mathrm{c}}\right)\end{array}$ & & & & $0.6 \pm 0.1$ & & \\
\hline & 2 & 17.15 & $0.25 \pm 0.03$ & & & & $0.6 \pm 0.1$ & & \\
\hline & 3 & 17.38 & $0.25+0.04$ & & & & & & \\
\hline & 4 & 17.60 & $0.24 \pm 0.04$ & & & & & & \\
\hline & 5 & 17.81 & $0.35 \pm 0.05$ & & & & & & \\
\hline & b & 18.02 & $0.31 \pm 0.09$ & & & & & & \\
\hline \multirow[t]{2}{*}{$B^{2} \Sigma_{u}^{+}$} & 0 & 18.76 & $\begin{array}{l}1.29 \pm 0.04 \\
\left(1.25,{ }^{2} 1.25,{ }^{b} 1.30^{c}\right)\end{array}$ & $-0.861^{\mathrm{e}}$ & & & & & \\
\hline & 1 & 19.04 & $\begin{array}{l}1.25 \pm 0.04 \\
\left(1.27^{b}\right)\end{array}$ & & & & & & \\
\hline
\end{tabular}

${ }^{2}$ Reference 4.

Beference 5 .

cReference 6.

dReference 25 .

values for the $v^{\prime}=0$ and $v^{\prime}=1$ peaks in the $584 \AA$ spectrum a re grossly different. In addition, we find a smaller although reproducible difference for the $537 \AA$ spectrum as well. The $\beta$ values measured for the $v^{\prime}=0$ peaks for 584 and $537 \AA$ ionization are identical, within experimental error. For the $v^{\prime}=2$ peak in the $584 \AA$ spectrum, we find a $B$ value significantly higher than that measured earlier by Morgenstern. ${ }^{5}$

Results obtained using the Ner discharge agree much better with those of Morgenstern ${ }^{5}$ than with those of Carlson。 ${ }^{4}$ The $\beta$ value obtained for $v^{\prime}=0$ at $627 \AA$ is significantly higher than at the other wavelengths studied and comparable to that obtained for the $v^{\prime}=1,2$ levels in the $584 \AA$ spectrum.

\section{B. $A^{2} \Pi_{u}$ band}

For the $584 \AA$ spectrum, we find a previously undetected difference in the values of $\beta$ for the first several members of the vibrational progression. For the $537 \AA$ photoionization, $\beta$ values are significantly higher than at $584 \AA$. The electron energy for the $v^{\prime}=0$ peak in the $736 \AA$ spectrum is exceedingly low, $0.16 \mathrm{eV}$. Angular distributions for such low electron energies are easily affected by stray electric and magnetic fields, which are responsible for the large error bars in the corresponding $\beta$. The result is nevertheless reliable.

\section{C. $B^{2} \Sigma_{u}^{+}$band}

The $\beta$ values at $584 \AA$ are in good agreement with those reported earlier. Because of the high value for the
${ }^{\mathbf{e}}$ Reference 14

${ }^{1}$ Reference 15.

${ }^{8}$ Reference 16 .

${ }^{\mathrm{h}}$ These results were the same for all vibrational levels reported.

ionization potential of this band, the 537 and $522 \AA$ spectra heavily overlap the $584 \AA$ spectrum of the $A^{2} \Pi_{u}$ band. For this reason we could not measure $\beta$ values for those higher order Her lines.

\section{DISCUSSION}

\section{A. Influence of autoionization}

The interpretation of the energy dependence of the $\beta$ values reported in this paper is influenced by the knowledge that the presence of autoionization can severely affect photoionization cross sections. As an example, autoionization has been invoked ${ }^{26}$ in the Ner $736 \AA$ and $744 \AA$ spectra of the $X^{2} \Sigma_{g}^{+}$band of $\mathrm{N}_{2}^{+}$, where the $v^{\prime}=2,3,4$ peaks have anomalously high vibrational intensities ${ }^{26}$ and low $\beta$ values ${ }^{5}$ relative to those of the $v^{\prime}=0,1$ peaks. Marr and Woodruff, ${ }^{27}$ using a synchroton radiation light source, have recently measured the branching ratio of the $X^{2} \Sigma_{g}^{*} v^{\prime}=0$ photoelectron peak (i.e., the ratio of the intensity of this peak to that of the whole band) and also found evidence for autoionization in a neighboring energy region. Thus, in order to interpret the present angular distribution data, we need to first assess the extent of autoionization for the range of photon wavelengths covered in this study.

Rydberg states are the dominant contributors to autoionization processes, ${ }^{28}$ through their interaction with the ionization continuum. This interaction is commonly described by the Fano theory of configuration interaction. ${ }^{28}$ Extensions of the theory by Mies $^{29}$ and Bardsley ${ }^{30}$ permitted Smith $^{31}$ to express vibrational intensities in 


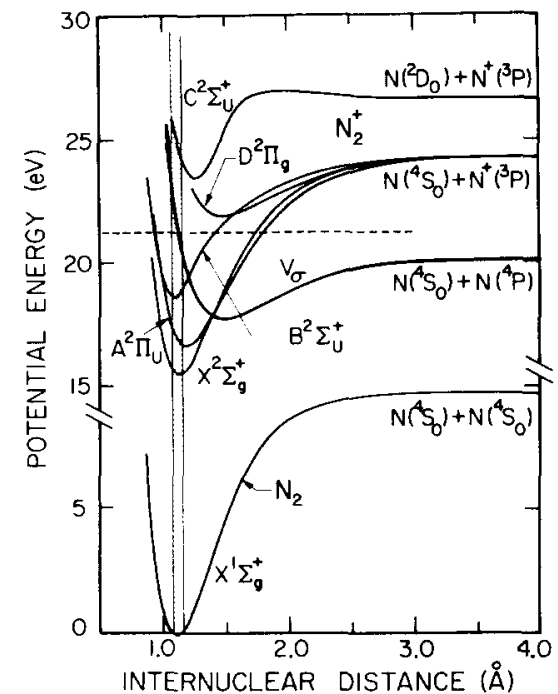

FIG. 5. Potential energy curve for the neutral autoionizing state, $V_{\sigma}$, and the low-lying ionic states of $\mathrm{N}_{2}$, after Gilmore. ${ }^{33}$ The dashed horizontal line lies at $21.21 \mathrm{eV}(584 \AA)$.

photoelectron spectra as the sum of two terms involving Franck-Condon factors, $F^{2}$ :

$$
I_{i v^{\prime}} \propto\left[F_{i v^{\prime}}^{2}+b_{i \nu} F_{i \nu}^{2} F_{\nu v^{\prime}}^{2}\right],
$$

where $i, \nu$, and $v^{\prime}$ index vibrational quantum numbers for initial, autoionizing, and final states, respectively, and $b_{i \nu}$ is a parameter. Equation (4) is valid only in two cases. $^{31}$ In the first, the energy width of the light source is small compared to the width of the resonance and the photon energy is tuned to the resonance maximum. In the second case, the energy width of the light source greatly exceeds the width of the resonance and the photon bandwidth encompasses the entire resonance.

In general, Franck-Condon factors between neutral states and ionic states for processes of direct ionization and autoionization appearing in Eq. (4) will be different, as Smith $^{31}$ and Kinsinger and Taylor ${ }^{32}$ have discussed for autoionization of molecular oxygen. The potential energy curves involved, and especially the corresponding equilibrium internuclear distances, were found to determine which vibrational peaks were enhanced. Potential energy curves for an ionic state and any Rydberg state converging to that ionic state would be similar. Thus, the Franck-Condon factors $F_{i \nu}^{2}$ corresponding to excitation to superexcited Rydberg states which converge to an ion should be approximately equal to the Franck-Condon factors $F_{i v}^{2}$, for direct photoionization to that ionic state. Potential energy curves ${ }^{33}$ for several of the states of $\mathrm{N}_{2}^{+}$to which we will constantly refer are shown in Fig. 5 .

In order to identify the Rydberg states which may contribute to an ionization process, it is convenient to estimate the positions of the corresponding energy levels. Rydberg electrons do not participate heavily in molecular bonding. We can, therefore, calculate their energies from the energy levels of the ion to which they converge and from transferable term values, $T(n, l, \lambda)$, by:

$$
E(\nu ; n, l, \lambda)=I_{n}(\nu)-T(n, l, \lambda) .
$$

Here $I_{b}(\nu)$ is the ionization potential for ions in electronic state $b$ and in vibrational state $\nu$. Term values $T(n, l$, $\lambda)$ a re assumed to depend only on the principal quantum number $n$ of the series $(l, \lambda)$, where $l$ specifies the electron angular momentum and $\lambda$ its projection onto the internuclear axis.

In order to assess the importance of autoionization, it is useful to try to obtain the ratio $Q_{v^{\prime}}^{\text {auto }} / Q_{v^{\prime}}^{\text {dir }}$ of the contribution of autoionization and direct ionization to the intensity of the different vibrational lines of a given photoelectron band. A knowledge of this ratio, together with Eq. (3), permits one furthermore to obtain a relation between $\beta_{\mathrm{dir}}$ and $\beta_{\text {auto }}$ from which we can determine one of them if the other is known. This ratio can be obtained as follows.

Let $r^{\prime}$ and $v^{\prime}$ denote the vibrational quantum numbers of two lines in a single band in the photoelectron spectrum of $\mathrm{N}_{2}$. It can be shown in a straightforward manner that:

$$
\frac{Q_{v^{\prime}}}{Q_{r^{\prime}}}=\frac{F_{i v^{\prime}}^{2}}{F_{i r^{\prime}}^{2}} \cdot \frac{1+\left(Q_{v^{\prime}}^{\text {ato }} / Q_{v^{\prime}}^{\text {dir }}\right)}{1+\left(Q_{r^{\prime}}^{\text {ato }} / Q_{r^{\prime}}^{\text {dir }}\right)},
$$

where $Q_{v^{\prime}}$ and $Q_{r^{\prime}}$ refer to the total cross sections corresponding to the lines being considered, and $F_{i v^{\prime}}^{2}$ and $F_{i r^{\prime}}^{2}$ are the associated Franck-Condon factors for direct vertical transitions from the ground state of the neutral molecule. The Franck-Condon ratio $F_{i v^{\circ}}^{2} / F_{i r^{\prime}}^{2}$ is independent of the incident wavelength, whereas the $Q_{v^{\prime}}^{\text {ayto }}$ $Q_{v^{\prime}}^{\text {dir }}$ and $Q_{r^{\prime}}^{\text {auto }} / Q_{r^{\prime}}^{\text {dir }}$ ratios a re not. Therefore, variation of $Q_{v^{\prime}} / Q_{r^{\prime}}$ with wavelength is indicative of the existence of autoionization.

The $Q_{v^{\prime}}^{\text {auto }} / Q_{v^{\prime}}^{\text {dir }}$ and $Q_{r^{\prime}}^{\text {auto }} / Q_{r^{\prime}}^{\text {dir }}$ ratios a re simply related to each other by appropriate Franck-Condon factors according to

$$
\frac{Q_{v^{\prime}}^{\text {ayto }} / Q_{v^{\prime}}^{\text {dir }}}{Q_{r^{\prime}}^{\text {2uto }} / Q_{r^{\prime}}^{d i r}}=\frac{F_{v v^{\prime}}^{2} / F_{\nu r^{\prime}}^{2}}{F_{i v^{\prime}}^{2} / F_{i r^{\prime}}^{2}},
$$

where, as mentioned above, $\nu$ denotes the intermediate neutral state through which the autoionization proceeds. Replacement of this into Eq. (6) furnishes

$$
\frac{Q_{v^{\prime}}^{\text {auto }}}{Q_{v^{\prime}}^{\text {dir }}}=\frac{\left(F_{i r^{\prime}}^{2} / F_{i v^{\prime}}^{2}\right)\left(Q_{v^{\prime}} / Q_{r^{\prime}}\right)-1}{1-\left(F_{v r^{\prime}}^{2} / F_{\nu v^{\prime}}^{2}\right)\left(Q_{v^{\prime}} / Q_{r^{\prime}}\right)} .
$$

This equation permits a determination of $Q_{v^{\prime}}^{\text {auto }} / Q_{v^{\prime}}^{\text {dir }}$ from a knowledge of the corresponding experimental total cross section ratio $Q_{v^{\prime}} / Q_{r^{\prime}}$ and appropriate FranckCondon factors.

Autoionization propensities depend on parameters of the electronic state involved as well. Bardsley ${ }^{34}$ has shown that the autoionization lifetimes of superexcited neutral states within a Rydberg series increase as $n^{3}$, where $n$ is the principal quantum number. Thus, the dominant contribution to vibrational peak enhancement will be made by autoionization of lower members of a Rydberg series.

The remarks just made will facilitate the assessment of the contribution of autoionization to the several bands 
in the photoelectron spectrum in $\mathrm{N}_{2}$ as indicated in the following sections.

\section{B. $A^{2} \Pi_{u}$ band}

We can infer the variation of $\beta$ with electron energy for the $A^{2} \Pi_{u}$ band, at several of the photon energies used in these studies, from the variation of $\beta$ across a vibrational progression as long as autoionization does not contribute to the ionization process. We should be able to detect the presence of autoionization or other processes competing with direct autoionization on the basis of the vibrational peak intensities for the band.

Gardner and Samson ${ }^{3}$ have measured vibrational peak intensities in the $584 \AA$ spectrum of the $A^{2} \Pi_{u}$ band, corrected for analyzer electron energy discrimination, and compared results to calculated Franck-Condon factors. They find reasonable agreement between theory and experiment, from which we infer that autoionization should not contribute significantly to the observed intensities. Vibrational peak intensities measured for the $537 \AA$ spectrum do not differ noticeably from those for the $584 \AA$ spectrum. Thus, the $\beta$ values obtained for the $537 \AA$ spectrum are probably not affected by autoioniza tion either.

Plummer et al. ${ }^{35}$ measured the partial photoionization cross section for the $A^{2} \Pi_{u}$ state of $\mathrm{N}_{2}$ using synchrotron radiation. That work evaluated the influence of autoionization using constant ion state (CIS) spectra, whereby the energy of the photoelectron is swept together with the photon energy. Structure in the CIS spectrum for the $A^{2} \Pi_{u}$ state shows autoionization to occur in the wavelength region from 740 to $680 \AA$.

Let us assess the possible contribution of autoionization levels to the $v^{\prime}=0$ peak of the $A^{2} \Pi_{u}$ band in the 736 $\AA$ spectrum. Table III contains the possible Rydberg state energies in the vicinity of $16.85 \mathrm{eV}$. These energies were calculated from the expression

$$
T(n, l, \lambda)=\frac{\mathrm{Ry}}{[n-\delta(l, \lambda)]^{2}},
$$

and Eq. (5). Ry is the Rydberg (13.606 eV) and the quantum defect $\delta$ was obtained from Table I of Ref. 36.
Since $\delta$ can vary with $n$ by several hundredths or more, and since it was assumed to be constant in these calculations, the accuracy of the numbers around $16.85 \mathrm{eV}$ in Table III is probably no better than about $0.02 \mathrm{eV}$. We might consider that the autoionizing Rydberg state incorporates a $B^{2} \Sigma_{u}^{+}$ionic core and a Rydberg orbital, since the ionization energy for the $\sigma_{u} 2 s$ orbital is 18.76 $\mathrm{eV}$. Optically allowed autoionizing states would need to incorporate $n s \sigma_{g}$ or $n d \lambda_{g}(\lambda=\sigma, \pi, \delta)$ Rydberg orbitals. Franck-Condon factors from ground neutral $\mathrm{N}_{2}$ to the various vibrational levels of the $B^{2} \Sigma_{u}^{+}$ion determine the extent of autoionization of Rydberg states incorporating the $B^{2} \Sigma_{u}^{+}$ion core and having the same degree of vibrational excitation as the ion. Intensities to $B^{2} \Sigma_{u}^{+}$vibrational states for $v^{\prime} \geqslant 1$ are small, so that autoionization from Rydberg states with $\nu \geqslant 1$ and incorporating the $B^{2} \Sigma_{u}^{+}$ion core can safely be neglected. In a consideration of Rydberg states with $\nu=0$, it was found that in no case do term values ${ }^{36}$ for the only experimentally known strong series $\left(n d \pi_{g}\right)$ give resulting energies [from Eq. (5)] within $100 \mathrm{meV}$ of 16.85 or $16.67 \mathrm{eV}$. Thus electronic autoionization of Rydberg states incorporating the $B^{2} \Sigma_{u}^{*}$ ionic core does not contribute to the intensity of any of the peaks, much less the $A^{2} \Pi_{u} v^{\prime}=0$ one, in the $736 \AA$ photoelectron spectrum. Autoionizing states more likely incorporate the $A^{2} \Pi_{u}$ ionic core.

If autoionization contributes to the intensity of the $A^{2} \Pi_{u} v^{\prime}=0$ peak in the $736 \AA$ spectrum, the only possible mechanism is vibrational autoionization. Here, the energy in excess of an ionization potential of a particular ionic vibrational level is supplied by a higher degree of vibrational excitation in the autoionizing Rydberg state. Plummer et al. ${ }^{35}$ have attributed the structure in their CIS spectrum around $736 \AA$ to vibrational autoionization of the $5 d \sigma_{g} v=3$ Rydberg state, but the resolution of their measurement makes it difficult to ascertain the exact location and extent of this autoionization. In the high resolution photoionization mass spectrometry study of Berkowitz and Chupka, ${ }^{37}$ no evidence for vibrational autoionization was detected for $\mathrm{N}_{2}$. Indeed, they confirm the presence of vibrational autoionization only in $\mathrm{H}_{2}$, where it had been previously predicted to occur. We conclude that the $A^{2} \Pi_{u}$ peak in the $736 \AA$ photoelectron

TABLE III. Predicted Rydberg state energy levels. a

\begin{tabular}{|c|c|c|c|c|c|c|c|c|c|}
\hline \multirow{2}{*}{$\begin{array}{l}\text { Ion } \\
\text { core }\end{array}$} & \multirow{2}{*}{$\begin{array}{l}\text { Vibrational } \\
\text { quantum } \\
\text { number }\end{array}$} & \multirow[b]{2}{*}{ Series } & \multirow{2}{*}{$\begin{array}{l}\text { I. P. of } \\
\text { ion }(e V)\end{array}$} & \multicolumn{6}{|c|}{ principal quantum number } \\
\hline & & & & $n=3$ & $n=4$ & $n=5$ & $n=6$ & $n=7$ & $n=8$ \\
\hline \multirow[t]{10}{*}{$A^{2} \Pi_{u}$} & 1 & $s \sigma_{g}$ & 16.92 & 13.38 & 15.37 & 16.05 & 16.37 & 16.54 & 16.64 \\
\hline & 1 & $d \sigma_{g}$ & 16.92 & 15.21 & 16.00 & 16.34 & 16.52 & 16.63 & 16.70 \\
\hline & 2 & $s \sigma_{g}$ & 17.14 & 13.60 & 15.59 & 16.27 & 16.59 & 16.76 & 16.86 \\
\hline & 2 & $d \sigma_{g}$ & 17.14 & 15.43 & 16.22 & 16.56 & 16.74 & $\underline{16.85}$ & 16.92 \\
\hline & 3 & $s \sigma_{g}$ & 17.37 & 13.83 & 15.82 & 16.50 & 16.82 & 16.99 & 17.09 \\
\hline & 3 & $d \sigma_{g}$ & 17.37 & 15.66 & 16.45 & 16.79 & 16.97 & 17.08 & 17.15 \\
\hline & 4 & $s \sigma_{g}$ & 17.59 & 14.05 & 16.04 & 16.72 & 17.04 & 17.21 & 17.31 \\
\hline & 4 & $d \sigma_{g}$ & 17.59 & 15.88 & 16.66 & 17.00 & 17.19 & 17.30 & 17.37 \\
\hline & 5 & $s \sigma_{g}$ & 17.82 & 14.28 & $\overline{16.27}$ & 16.95 & 17.27 & 17.44 & 17.54 \\
\hline & 5 & $d \sigma_{z}$ & 17.82 & 16.11 & 16.89 & 17.23 & 17.42 & 17.53 & 17.60 \\
\hline$B^{2} \Sigma_{u}^{+}$ & 0 & $d \sigma_{g}$ & 18.76 & 17.18 & 17.88 & 18.19 & & & \\
\hline
\end{tabular}

${ }^{2} U s i n g$ the following quantum defects from Ref. $36 ; s \sigma_{g}, 1.04 ; d \sigma_{g}, 0.18 ; d \pi_{g}, 0.07$. 


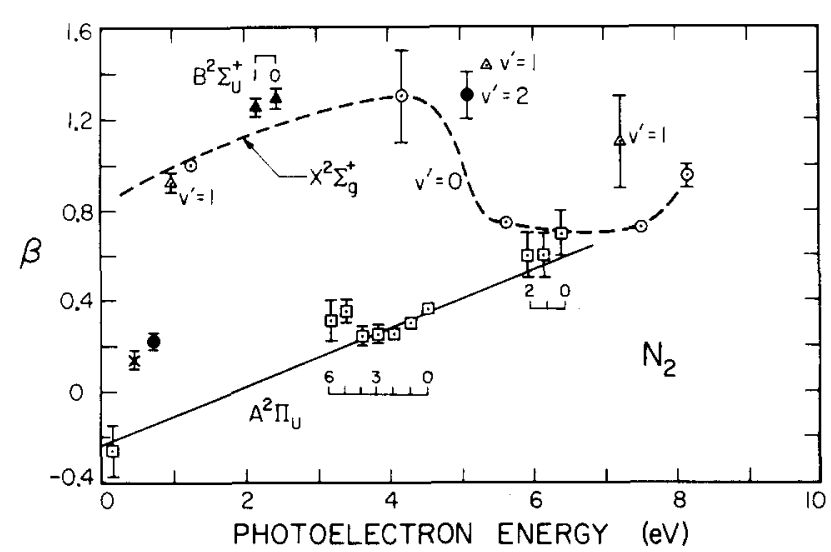

FIG. 6. Plot of experimental $\beta$ values versus electron energy for $\mathrm{N}_{2}$ ionized by $736,627,584,537$, and $522 \AA$ light. $\odot, v^{\prime}$ $=0,{ }^{2} \Sigma_{g}^{+} ; \Delta, v^{\prime}=1,{ }^{2} \Sigma_{g}^{+} ; \bullet, v^{\prime}=1,{ }^{2} \Sigma_{g}^{+} ; x, v^{\prime}=3,{ }^{2} \Sigma_{g}^{+} ; \square$, peaks of the $A^{2} \Pi_{u}$ state. $\triangle$, peaks of the $B^{2} \Sigma_{u}^{*}$ state. Solid line represents variation of $\beta$ for vibrational peaks of the $A^{2} \Pi_{u}$ band for experiments at three photon energies. Dashed line represents variation of $\beta$ for the $v^{\prime}=0$ peak of the $X^{2} \Sigma_{\xi}^{*}$ band for experiments at five photon energies.

spectrum is probably not significantly contaminated by electronic or vibrational autoionization.

In summary, autoionization seems not to be an important contribution to any of the vibrational peaks in the $A^{2} \dot{\Pi}_{u}$ band at any of the photon wavelengths of 584 , 537 , and $736 \AA$. As a result, the variation of $\beta$ with vibrational quantum number $v^{\prime}$ for this band can be interpreted as due to the effect of photoelectron energy. In Fig. 6 we have plotted all the values of $\beta$ for the vibrational peaks of the $A^{2} \Pi_{u}$ band which were measured, as well as those for the $X^{2} \Sigma_{g}^{+}$and $B^{2} \Sigma_{u}^{+}$bands. The $A^{2} \Pi_{u}$ data points seem to fall on a single curve, represented approximately by the solid line of that figure. This is consistent with the interpretation that this line represents the variation of $\beta$ with electron energy for the $A^{2} \Pi_{u}$ ionic state over the photoelectron energy range $0-6.3 \mathrm{eV}$. The value of $\beta$ for this band increases from a value close to -0.24 at threshold at a rate of roughly $+0.13 \mathrm{eV}^{-1}$ over that energy range. This energy dependence is reminiscent of the one calculated by Manson ${ }^{12}$ for carbon $2 p$ electrons in this same energy range. The experimental $\beta$ values for the ionization of electrons in olefins ${ }^{32,38,39}$ have an analogous behavior.

\section{C. $X^{2} \Sigma_{g}^{+}$band}

The measurements of $\beta$ for this band were made for a $7 \mathrm{eV}$ photoelectron energy range which is wide enough for trends due to possible contributions of autoionization to be clearly visible. The corresponding points are plotted in Fig. 6. The dashed line in that figure connects the points for the $v^{\prime}=0$ peak of the $X^{2} \Sigma_{g}^{+}$band and shows an abrupt shift in $\beta$ at an electron energy near $4.5 \mathrm{eV}$. On the other hand, the $v^{\prime}=1$ points for 736 and $584 \AA$ and the $v^{\prime}=2$ point for $584 \AA$ lie along a line which can be obtained by smooth extrapolation to higher energies of the low energy portion $(<4 \mathrm{eV})$ of the $v^{\prime}=0$ dashed curve. This indicates that something special is occurring for $v^{\prime}=0$ at photoelectron energies above 4.5
eV. We now discuss possible explanations for this effect.

\section{Availab/e autoionizing states}

The role of autoionization can be investigated, based upon a knowledge of potential enery curves for the ionic and high-lying Rydberg states. Autoionization of $\mathrm{N}_{2}$ above $15.58 \mathrm{eV}$ has been studied by the techniques of photoelectron spectroscopy, ${ }^{26}$ photoionization mass spectroscopy, ${ }^{37}$ and optical absorption spectroscopy. ${ }^{40-42}$ Let us now consider the possible presence of states which autoionize to the $\mathrm{N}_{2}^{+} X^{2} \Sigma_{g}^{+}$in three ranges of incident photon energy: low energy Ner discharge lines (16.67 and 16.85 eV), high energy Ner discharge lines $(\sim 19.78 \mathrm{eV})$, and $\mathrm{HeI}$ discharge lines $(21.22,23.09$, and $23.74 \mathrm{eV})$.

Several Rydberg states are available in the low energy NeI discharge line region (736 and $744 \AA$ ). Indeed, values of the excitation energies calculated from $\mathrm{Eq}$. (5) and presented in Table III were earlier used in the discussion of autoionization to the $A^{2} \Pi_{u}$ ionic state. Since the Franck-Condon factors for direct photoionization are small for $v^{\prime} \geqslant 5$ (as can be seen from Fig. 2), so are the $F_{i \nu}^{2}$ for $\nu \geqslant 5$, and excitation to the corresponding Rydberg states can be neglected. We note that $E(\nu=4$; $\left.4 d \sigma_{g}\right)=16.66 \mathrm{eV}$ is equal to the energy of the second strongest Net emission line which lies at $743.7 \AA$. This energy corresponds well to the peak in the photoionization spectrum of Berkowitz and Chupka ${ }^{37}$ which lies at $743.5 \AA$. We also calculate that $E\left(\nu=2 ; 7 d \sigma_{s}\right)=16.85 \mathrm{eV}$ is equal to the energy of the $736 \AA$ Ner line.

The energies of other autoionizing Rydberg states, $E\left(\nu=3 ; 5 d \sigma_{g}\right)=16.79 \mathrm{eV}, E\left(\nu=3 ; 6 s \sigma_{g}\right)=16.82 \mathrm{eV}$, and $E\left(\nu=2 ; 8 s \sigma_{g}\right)=16.86 \mathrm{eV}$, lie nearby. Thus these states may contribute somewhat to the autoionization since the width of the autoionizing lines ${ }^{37}$ in this region of the spectrum is of the order of $0.02 \mathrm{eV}$, and the error in using Eq. (9) around this photon energy is also of the order of $0.02 \mathrm{eV}$. We conclude that contributions to autoionization should result from $\nu=2, \nu=3$, or both.

For experiments at $627 \AA$, there seems to be no possibility of autoionization. Indeed, the corresponding energy, $19.78 \mathrm{eV}$, lies well above all Rydberg states which converge to $v^{\prime}=0$ and $v^{\prime}=1$ of the $\mathrm{N}_{2}^{+} B^{2} \Sigma_{u}^{+}$state (18.76 and $19.05 \mathrm{eV}$, respectively). Furthermore, no Rydberg states with $r_{e}$ close to that of $\mathrm{N}_{2}^{+2} \Sigma_{g}^{+}$or $\mathrm{N}_{2}{ }^{1} \Sigma_{z}^{+}(\sim 1.1 \mathrm{~A})$, converging to higher ionization potentials, are known in this region. Indeed, the absorption cross section curves of Lee et al. ${ }^{40}$, Sasanuma et al. , ,1 and Watson et al. ${ }^{42}$ are remarkably structureless; Rydberg states converging to the $C^{2} \Sigma_{u}^{*}$ ion are not found spectroscopically for $\lambda>550 \AA .{ }^{43}$ There is a possibility that neutral Rydberg states exist which converge to the $D^{2} \Pi_{g}$ ion. In this case, the potential energy curves are expected to be similar to those of the $D^{2} \Pi_{s}$ ion, which has a large $r_{e}$ $(1.471 \AA) .{ }^{44}$ As a result, Franck-Condon factors to bound vibrational levels in this potential well are calculated to be exceedingly low. ${ }^{45}$ Vertical transitions from ground neutral $\mathrm{N}_{2}$ to these Rydberg states necessarily intersect the potential energy curves above the dissocia- 
tion limit, $20.089 \mathrm{eV}$ (corresponding to $N^{4} S_{0}+N^{4} P$ ) which lies above the $19.78 \mathrm{eV}$ photon energy. Finally, the synchrotron radiation $X^{2} \Sigma_{g}^{+} v^{\prime}=0$ vibrational branch ing ratio measurements of Marr and Woodruff near 20 $\mathrm{eV}$ photon energy are remarkably wavelength independent. This reinforces the conclusion that only direct photoionization occurs at $627 \AA$ 。

For photon energies above $20.089 \mathrm{eV}$, as for all of the Her lines, the possibility of autoionization cannot be eliminated. Carlson ${ }^{4}$ suggested earlier that no neutral states lie at $21.22 \mathrm{eV}$ (i.e., $584 \AA$ ), based on three a rguments. First, resonance absorption via Rydberg states converging to the ionic state at $37.3 \mathrm{eV}$ (which corresponds to ionization of a $\sigma_{g} 2 s$ electron) should not be possible at the relatively low photon energy of 21.22 eV. Second, Samson and Cairns ${ }^{46}$ observed no resonance absorption at $584 \AA$ in their absorption experiment. Third, absorption to Rydberg states below ions $C^{2} \Sigma_{u}^{+}$and $D^{2} \Pi_{g}$ are conceivable, but less probable because of the double excitation nature of the process needed to form them. This process involves the promotion of one bonding electron to an antibonding $\sigma_{u} 2 p$ or $\pi_{g} 2 p$ orbital and the ejection of another bonding electron.

Let us examine Carlson's suggestion ${ }^{4}$ in greater detail. His first and second arguments above assume that the autoionizing state is a discrete vibrational level of an electronic state resonant with the $584 \AA$ Her radiation. This assumption is not necessarily correct. Indeed, as we have just suggested, the potential energy curves for neutrals in the 617 to $550 \AA$ wavelength region are repulsive over the range of internuclear distances accessible from the ground state by vertical transitions, and lie above the dissociation limit into $N\left({ }^{4} S_{0}\right)+N\left({ }^{4} P\right)$. Therefore, nuclear continuum autoionizing states can still exist in this wavelength region, which encompasses the $584 \AA$ excitation line under consideration.

The argument that multiple excitation processes are less probable is fundamentally sound, but we will argue that they are not negligible. Indeed, in the $304 \AA$ photoelectron spectra of $\mathrm{N}_{2}$, several peaks are seen which correspond to double excitation processes. ${ }^{21-23}$ The peaks are substantially weaker than those corresponding to excitation to the $X^{2} \Sigma_{g}^{+}, A^{2} \Pi_{u}$, or $B^{2} \Sigma_{u}^{+}$ionic states. Production rates for double excitation neutrals, where one electron is promoted to a higher valence orbital and another to a Rydberg orbital, have not been as

TABLE IV. Meinel band Franck-Condon factors. ${ }^{a}$

\begin{tabular}{lccccc}
\hline \hline $\begin{array}{l}\text { Vibrational } \\
\text { level } \nu \text { of } \\
A^{2} \Pi_{u}\end{array}$ & 0 & 1 & 2 & 3 & 4 \\
\hline 1 & 0.3255 & 0.0312 & 0.3358 & 0.2368 & 0.0624 \\
2 & 0.1360 & 0.2245 & 0.0214 & 0.1851 & 0.2946 \\
3 & 0.0453 & 0.1990 & 0.0797 & 0.1048 & 0.0627 \\
4 & 0.0133 & 0.1032 & 0.1745 & 0.0072 & 0.1553 \\
5 & 0.0036 & 0.0414 & 0.1395 & 0.1078 & 0.0069 \\
\hline \hline
\end{tabular}

aReference 54 .
TABLE V. Relative vibrational peak intensities ${ }^{\mathbf{a}}$ and calculated direct ionization Franck-Condon factors for the $X^{2} \Sigma_{g}^{+}$band.

\begin{tabular}{llc}
\hline \hline Peak & Experimental $^{\mathrm{b}}$ & $\begin{array}{l}\text { Franck-Condon } \\
\text { factor }\end{array}$ \\
\hline 0 & 100.0 & 100.0 \\
1 & $6.94 \pm 0.66$ & $9.27^{\mathrm{c}}$ \\
2 & $0.32 \pm 0.21$ & $0.59^{\mathrm{c}}$ \\
3 & $\cdots$ & $0.05^{\mathrm{d}}$ \\
\hline
\end{tabular}

${ }^{a}$ Normalized to 100.0 at $v^{\prime}=0$.

bMeasured at $584 \AA$, Ref. 3 .

${ }^{c}$ Corrected for variation of transition moment with internuclear distance $r$, Ref. 3 .

Not corrected for variation of transition moment with $r$, Ref. 45 .

well characterized. Electron ${ }^{47-50}$ and photon ${ }^{51,52}$ impact fluorescence studies have detected a $1200 \AA$ atomic emission $\left({ }^{4} P-{ }^{4} S_{0}\right)$ having a threshold impact energy of $20.089 \mathrm{eV}$. Aarts and de Heer ${ }^{47}$ have suggested that Rydberg states lying below the $D^{2} \Pi_{g}$ ion are probably responsible for this emission. Lee et al., ${ }^{51}$ in a photon impact study, measured an atomic fluorescence cross section of $2 \times 10^{-19} \mathrm{~cm}^{2}$ for an incident light wavelength near $584 \AA$. The partial photoionization cross section for the production of $\mathrm{N}_{2}^{+} X^{2} \Sigma_{s}^{+}$in all vibrational levels is $8.4 \times 10^{-18} \mathrm{~cm}^{2}$ at $584 \AA$, as measured by Samson et al. ${ }^{53}$ Therefore, the atomic fluorescence cross section, arising from double electron excitations to neutral states, is about $2.4 \%$ of the $X^{2} \Sigma_{g}^{*}$ photoionization cross section, a small but non-negligible fraction. Conceivably, the same neutral states could autoionize in competition with the dissociative process. Indeed, for $\mathrm{N}_{2}$, the autoionization lifetime for autoionizing Rydberg states is probably $n^{3} \times 10^{-14} \mathrm{sec}^{34}$ where $n$ is an effective principal quantum number. On the other hand, dissociation occurs on a time scale of $10^{-12}-10^{-13}$ sec. Therefore, autoionization from the lower $(n=3,4)$ Rydberg states can occur before dissociation.

We conclude that autoionizing neutral states can exist in the energy region near 16.8 and $21.2 \mathrm{eV}$ but not near $19.8 \mathrm{eV}$. The mechanism for autoionization of the states near $21.2 \mathrm{eV}$ is probably different than for those states formed by 736 and $744 \AA$ light, as discussed in Sec. V。C4。

\section{Extent of autoionization}

In the $736 \AA$ spectrum, we can calculate the relative enhancement of the various vibrational peaks from the above assessment of which vibrational levels may be responsible for the electronic autoionization and from the use of Eq. (8). The appropriate Franck-Condon factors, $F_{v v^{\prime}}^{2}$, from the neutral autoionizing state to the ionic state, correspond to the Meinel band emission $\left(A^{2} \Pi_{u}-X^{2} \Sigma_{g}^{+}\right)$. They have been tabulated by Nicholls ${ }^{54}$ and are listed in part in Table IV. The calculated Franck-Condon factors for the $X^{1} \Sigma_{g}^{+} \rightarrow X^{2} \Sigma_{g}^{+}$direct ionization are given in the last column of Table $V_{0}$. From these sets of Franck-Condon factors and the $Q_{v^{\prime}} / Q_{r^{\prime}}$ which can be obtained from the ratio of peak intensities 
TABLE VI. Relative contribution of autoionization to the intensities of the vibrational peaks of the $X^{2} \Sigma_{g}^{+}$band at $736 \AA$.

\begin{tabular}{llllll}
\hline & & \multicolumn{5}{c}{$v^{\prime}$} \\
& $\nu$ & 0 & 1 & 2 & 3 \\
\hline$Q_{v^{\prime}}^{\text {ato }} / Q_{v^{\prime}}^{\text {dir }}$ & 2 & 0.064 & 0.42 & $1.1 \times 10^{2}$ & $1.7 \times 10^{2}$ \\
& 3 & 0.036 & 0.40 & 22 & $1.7 \times 10^{2}$ \\
$Q_{v^{\prime}}^{\text {ato }} /\left(Q_{v^{\prime}}^{\text {ato }}+Q_{v^{\prime}}^{\text {dir }}\right)$ & 2 & 0.060 & 0.30 & 0.991 & 0.994 \\
\hline \hline
\end{tabular}

in Fig. 3 it is possible, using Eq. (8), to calculate the contribution from autoionization to each of the peaks for which $v^{\prime} \neq r^{\prime}$ in the $X^{2} \Sigma_{g}^{+} 736 \AA$ spectrum once a value of $\nu$ is assumed and a reference $r^{\prime}$ is chosen. As discussed in Sec. V.C 1 , the appropriate assumptions are $\nu=2$ and $3 .{ }^{55}$ Choosing in addition $r^{\prime}=0$, we obtain the $Q_{v^{\prime}} / Q_{r^{\prime}}$ for $v^{\prime}=1,2$, and 3 given in Table VI. The value for $v^{\prime}=r^{\prime}=0$ can then be obtained from Eq. (7) using the values for one of the $v^{\prime} \neq r^{\prime}$. We have chosen $v^{\prime}=3$ for that purpose. A choice of $v^{\prime}=1$ or 2 gives comparable results; the choice of $v^{\prime}=1$ also gives a value for $Q_{0}^{\text {auto }} /$ $Q_{0}^{\text {dir }}$ consistent with the previous four results. However, for $v^{\prime}=2$ this quantity becomes about two orders of magnitude larger mainly because of the anomalously small value of $F_{22}^{2} / F_{20}^{2}$ which results from the very small value of $F_{22}^{2}$ in Table VI. This indicates a certain amount of inconsistency in the values of Table VI which may arise from the fact that anomalous Meinel band Franck-Condon factors, which refer to ion $\rightarrow$ ion transitions, may not be completely appropriate to describe the neutral Rydberg $\rightarrow$ ion transitions being considered here. It nevertheless seems reasonable to conclude that estiestimates of $Q_{v^{\prime}}^{\text {2uto }} /\left(Q_{v}^{\text {2uto }}+Q_{v^{\prime}}^{\text {dir }}\right)$ given in Table VI are of the correct order of magnitude and therefore that autoionization contributes little to the intensity of the $v^{\prime}=0$ peak of the $X^{2} \Sigma_{z}^{+}$band at $736 \check{\mathrm{A}}$, moderately to the $v^{\prime}=1$ peak and dominantly to the $v^{\prime}=2$ and 3 peaks. As a self-consistency argument, we can use the $Q_{0}^{\text {auto }} /\left(Q_{0}^{\text {anto }}+Q_{0}^{\text {dir }}\right)$ of Table VI together with the calculated direct ionization Franck-Condon factors of Table $V$ and the experimental peak intensities of Fig. 3 to recalculate $Q_{v^{\prime}}^{\text {auto }} /\left(Q_{v^{\prime}}^{\text {auto }}+Q_{v^{\prime}}^{\text {dir }}\right)$ for $v^{\prime}=1$ through 3 without the use of the Meinel band Franck-Condon factors of Table IV. The results obtained are, respectively, 0.33 $\left(v^{\prime}=1\right), 0.96\left(v^{\prime}=2\right)$, and $0.994\left(v^{\prime}=3\right)$ for $Q_{0}^{\text {auto }} /\left(Q_{0}^{\text {auto }}\right.$ $+Q_{0}^{\text {dir }}$ ) equal to 0.060 , and $0.31\left(v^{\prime}=1\right), 0.96\left(v^{\prime}=2\right)$, and $0.994\left(v^{\prime}=3\right)$ for $Q_{0}^{\text {auto }} /\left(Q_{0}^{\text {auto }}+Q_{0}^{\text {dir }}\right)$ equal to 0.035 , in excellent agreement with the values of Table VI. This self-consistency strengthens the conclusion that autoionization is responsible for only about $5 \%$ of the $v^{\prime}=0$ peak intensity of the $X^{2} \Sigma_{g}^{+}$band at $736 \AA$.

For the $584 \AA$ spectrum, the extent of autoionization in the $X^{2} \Sigma_{g}^{+}$band is easier to estimate. Brundle ${ }^{56}$ found that the ratio of the $v^{\prime}=1$ to $v^{\prime}=0$ peak intensities for this band at $\theta=90^{\circ}$ was 0.08 at $584 \AA$ and 0.13 at $304 \AA$. Since at $304 \AA$ the photon energy is too high to produce autoionization, Carlson ${ }^{4}$ inferred from these data that autoionization had to be responsible for at least $40 \%$ of the total $X^{2} \Sigma_{g}^{*}$ band intensity at $584 \AA$, but felt that this was an improbable requirement.

Let us reexamine this conclusion by trying to estimate $Q_{v^{\prime}}^{\text {auto }} / Q_{v^{\prime}}^{\text {dir }}$ for the two strongest peaks $\left(v^{\prime}=0,1\right)$ of the $X^{2} \Sigma_{g}^{+}$ionization produced by $584 \AA$ photons. For this purpose, let us consider the "trend line" determined by the low energy portion of the dashed curve in Figure VI. This curve goes through the points corresponding to ionization in the $v^{\prime}=0$ (by 736 and $627 \AA$ photons) and $v^{\prime}=1$ (by $736 \AA$ photons) levels of this state. We will show below that the contribution of autoionization to these points is small. Since $\beta$ for the $v^{\prime}=1$ peak at 584 $\AA$ lies along an extrapolation of this trend line, we infer that the intensity of this peak is not appreciably enhanced by autoionization. As a result, we may set in $\mathrm{Eq}$. (6) $r^{\prime}=1$ and $\left(Q_{1}^{\text {auto }} / Q_{1}^{\mathrm{d} 1 \mathrm{r}}\right)=0$ 。 Table $\mathrm{V}$, in addition to calculated Franck-Condon factors for direct ionization, contains normalized experimental peak intensities, corrected for electron energy analyzer discrimination effects. From the values in that table and $\mathrm{Eq}$. (6), we get $\left(Q_{0}^{\text {auto }} / Q_{0}^{d 1 r}\right)=0.33 \pm 0.13$. Since the $v^{\prime}=2$ peak is much weaker than the $v^{\prime}=0$ one, we can neglect its contribution to the total intensity of the $X^{2} \Sigma_{g}^{+}$band. From these numbers we conclude that $23 \pm 7 \%$ of the total $X^{2} \Sigma_{g}^{+}$band intensity can be attributed to autoionization. Plummer et al. ${ }^{35}$ estimated the contribution of autoionization at $584 \AA$ to the cross section for formation of the $X^{2} \Sigma_{g}^{+}$state to be $30 \%$ and of the $A^{2} \Pi_{u}$ to be $15 \%$.

We see no reason to consider our $23 \%$ figure, which is significantly lower than Carlson's $40 \%$ value, to be unacceptably high. Indeed, the intervening neutral autoionizing state is dissociative over the range of internuclear distances sampled by the ground state neutral Franck-Condon band (see Figure V), and there is therefore no need to require an accidental resonance at 584 $\AA$ with a discrete vibrational level. An additional test of whether autoionization has a prominent role in photoionization processes is the presence or absence of extra structure or intensity in the photoionization spectrum around the corresponding wavelength. The recent measurements of Samson et al. ${ }^{53}$ give the photoionization cross section to the $X^{2} \Sigma_{g}^{*}$ state by $584 \AA$ photons as approximately $8.4 \times 10^{-18} \mathrm{~cm}^{2}$ out of a total cross section at this wavelength of about $2.3 \times 10^{-17} \mathrm{~cm}^{2}$. One would conclude therefore that the $23 \%$ contribution of autoionization to the $X^{2} \Sigma_{\text {e }}^{*}$ band intensity corresponds to a contribution of $8.4 \%$ (i.e., $1.9 \times 10^{-18} \mathrm{~cm}^{2}$ ) to the total absorption cross section at $584 \AA$. Furthermore, the dissociative nature of the neutral ionizing state would suggest that this relatively small contribution need not be strongly wavelength dependent. These conclusions are therefore not inconsistent with the general flat and structureless nature of the absorption spectrum in the neighborhood of $584 \AA$, as mentioned in Sec. V.C1. The total absorption cross section measurements are in this case not sufficiently sensitive to detect processes which may compose a small fraction of the total cross section. Nevertheless, as we have just shown, such processes could significantly affect the corresponding $\beta$. We conclude that the measurements of $\beta$ can constitute a much more sensitive tool for detecting autoionization. 


\section{Determination of $\beta_{\text {auto }}$}

Considerations of vibrational intensity within a given photoelectron spectral band, as discussed above, give quantitative information about the extent of autoionization. With this information, application of Eq. (3) can furnish a relation between the quantities $\beta_{\text {auto }}$ and $\beta_{\text {dir }}$. These quantities can, in principle, also be calculated from quantum mechanics although such calculation is very difficult. ${ }^{12-17}$

Let us estimate $\beta_{\text {auto }}$ for the $X^{2} \Sigma_{g}^{+}$band at 736,627 , and $584 \AA$. For cases in which autoionization plays a dominant role and the autoionization lifetime exceeds the molecular rotational period, the photoelectron angular distribution should be isotropic (i.e., $\beta=0$ ). ${ }^{4}$ This effect predominates in the $736 \AA$ ionization of $\mathrm{N}_{2}$ for $v^{\prime}=2,3$ of the $X^{2} \Sigma_{g}^{+}$state reported here and previously, as well as in the $736 \AA$ ionization of $\mathrm{O}_{2}$ for $v^{\prime} \geq 4$ of the $X^{2} \Pi_{g}$ state reported previously. ${ }^{4,5}$

For experiments at $627 \AA$, unlike $736 \AA$, there seems to be no possibility of competing autoionization, as mentioned in Sec. V.C1. Therefore, $\beta_{d i r}$ equals the experimental $\beta$ for the corresponding photoelectron energy.

For photon energies above $20.089 \mathrm{eV}$, the threshold for dissociation of $\mathrm{N}_{2}$ into $N\left({ }^{4} S_{0}\right)+N\left({ }^{4} P\right)$, the possibility that autoionization might enhance vibrational peaks cannot be eliminated, as discussed in Sec. V.C1. Thus to obtain $\beta_{\text {auto }}$ for $\lambda=584 \AA$, let us first estimate $\beta_{\text {dir }}$ from a consideration of experiments done at other wavelengths. Roughly speaking, the peaks for $v^{\prime}=1,2$ produced by the $584 \AA$ line lie along the extrapolation of the results for $\lambda>584 \AA$ (see Fig. 6), whereas for $v^{\prime}=0$ it lies significantly below this trend line. We will assume that at $584 \AA$, the value of $\beta_{\mathrm{dir}}$ for $v^{\prime}=0$ is the same as for $v^{\prime}=1$ and equal to the experimental value of $\beta=1.43$ (see Table II) since as just indicated autoionization does not contribute to the latter. The experimental $\beta$ for $v^{\prime}=0$ at this wavelength is $0.74 \pm 0.02$. In Sec. V.C2 we showed that $Q_{0}^{\text {auto }} /\left(Q_{0}^{\text {auto }}+Q_{0}^{\text {dir }}\right)$ is equal to 0.25 at $584 \AA$. These values and Eq. (3) furnish $\beta_{\text {auto }}=-1.2 \pm 0.4$, significantly below 0 , but within the error bars of the permissible ${ }^{11}$ range of $\beta,-1 \leq \beta \leq 2$. This result may seem odd until we realize that the neutral autoionizing state may not persist for a full rotational period $\left(10^{-11} \mathrm{sec}\right)$. This is consistent with the argument presented in Sec. V.C1 according to which the autoionizing state involved should be dissociative. Therefore, for autoionization to compete with dissociation it must occur in a time of the order of or less than the dissociation time of about $10^{-12}$ to $10^{-13}$ sec. An alternate interpretation of $\beta_{\text {auto }}$ $=-1$ can be considered in the context of Dill's treatment of angular momentum transfers ${ }^{17}$ in photoionization. This theory was applied to the ionization of xenon, for which resonances in $\beta$ accompany resonances in the ionization cross section. In the cases of relatively longlived autoionizing states, at the extrema of the cross sections versus electron kinetic energy, the normally forbidden angular momentum transfers mix heavily with the allowed transfers. The asymmetry parameter for a forbidden angular momentum transfer is -1 .

\section{A quantitative model}

From the treatment above, we see that $\beta_{\text {auto }}$ depends on the type of autoionization process involved. For the usual type of autoionization occurring through a discrete neutral state, $\beta_{\text {auto }}$ is close to $0 .{ }^{4}$ For rapid autoionization through a dissociative state, the autoionization must occur very rapidly, as just discussed.

Let us consider a model for the variation of $\beta$ with photoelectron energy for the $v^{\prime}=0,1,2$ peaks of the $X^{2} \Sigma_{g}^{+}$band for photon energies greater than $20.089 \mathrm{eV}$, assuming, in agreement with the results of the previous section, that $\beta_{\text {auto }}=-1$ and $\beta_{\text {dir }}=1.43$. This model should also explain the enhancement of the $v^{\prime}=0$ peak of this band due to autoionization.

The model consists in (a) calculating $Q_{v^{\prime}}^{\text {auto }} / Q_{v^{\prime}}^{\text {dir }}$ from Eq. (4) (which assumes the validity of the Fano theory of configuration interaction ${ }^{28}$ ) and (b) replacing this in Eq. (3) using the values of $\beta_{\text {auto }}$ and $\beta_{\text {dir }}$ just mentioned. $Q_{v^{\prime}}^{\text {auto }} / Q_{v^{\prime}}^{\text {dir }}$ is [see Eq。(4)]

$$
\frac{Q_{v^{\prime}}^{\text {auto }}}{Q_{v^{\prime}}^{\text {dir }}}=b_{i \nu} \frac{F_{i \nu}^{2} F_{v v^{\prime}}^{2}}{F_{i v^{\prime}}^{2}} .
$$

The quantity $b_{i v}$ is independent of incident photon energy (and therefore of photoelectron kinetic energy) and of final vibrational quantum number and is determined by normalizing the theory to experiment for $v^{\prime}=0$ at $584 \AA$. $F_{i v^{\prime}}^{2}$ is also independent of photon energy and is taken from the calculated results of Table $V$. The continuumbound state Franck-Condon factors $F_{i v}^{2}$ and $F_{\nu v^{\prime}}^{2}$ are photon energy dependent and are calculated using momentum-normalized continuum wavefunctions.

In order to calculate the nuclear continuum wavefunctions needed for the $F_{i v}^{2}$ and $F_{\nu v^{\prime}}^{2}$ terms, we need to calculate the potential energy curve of the repulsive autoionizing state. A number of assumptions are necessary, since such states in the region above $20 \mathrm{eV}$ have not been well characterized. We can, nevertheless, infer the form of the potential energy curve based on anticipated symmetries, effects of the Rydberg series principal quantum number, similarities to known ionic states in the energy region, and dissociation limits. We next discuss some of those arguments.

Dipole selection rules from the ground to a Rydberg state of neutral $\mathrm{N}_{2}$ permit either $n p \sigma_{u}$ or $n p \pi_{u}$ Rydberg orbitals to be associated with the expected $D^{2} \Pi_{g}$ ion core. In this calculation we allowed absorption to the states having each of these two electronic configurations to occur with equal probability. Rates of electronic autoionization of neutral Rydberg states vary inversely with the cube of the effective principal quantum number. ${ }^{34} \mathrm{Cor}$ respondingly, we consider only the $3 p \sigma_{u}+$ core and $3 p \pi_{u}$ + core configurations. As a result of these two assumptions, we let the potential energy at the bottom of the $3 p \sigma_{u}+$ core and $3 p \pi_{u}+$ core potential wells lie, respectively, 3.90 and $4.16 \mathrm{eV}$ below that of the $D^{2} \Pi_{g}$ ion. The energy difference was chosen to agree with the difference between $3 p \sigma_{u}$ and $3 p \pi_{u}$ term values for the Rydberg series lying below the $X^{2} \Sigma_{g}^{+}$first ionization potential. ${ }^{36}$ The average of 3.90 and $4.16 \mathrm{eV}$ was chosen to result in sizeable values of $F_{i \nu}^{2} F_{\nu v^{\prime}}^{2}$ for $v^{\prime}=0$ and small values for $v^{\prime}=1,2$ for ionization by $584 \AA$ photons. 
Both potential energy curves were assumed to dissociate into $\mathrm{N}\left({ }^{4} S_{0}\right)+\mathrm{N}\left({ }^{4} P\right)$ atoms at $20.089 \mathrm{eV}$ photon energy, corresponding to the observed threshold for 1200 $\AA$ emission, ${ }^{4} P \rightarrow{ }^{4} S .{ }^{47-52}$ The $D^{2} \Pi_{g}$ ion is repulsive in the Franck-Condon region accessible from the ground $\mathrm{N}_{2}$ state, as is shown by the hatched region of Fig. 5. We require the Rydberg states which were thought to lie in a series which converged to the $D^{2} \Pi_{g}$ ion to be repulsive in the Franck-Condon region as well. The value of $r_{e}$ for the Rydberg states was taken to be equal to that of the $D^{2} \Pi_{g}$ state $\left.(1.471 \AA)\right)^{44}$ Further, we parameterized the potential energy for $r<r_{e}$ as a Morse potential with parameters fitted to the bottom of the RKR potential of the $D^{2} \Pi_{g}$ ion and to the corresponding classical turning points for $v=5$ and 10. For $r>r_{e}$, the Rydberg state potential energy curves were taken to be of Morse form, where the dissociation energy and range parameter were different from those for $r<r_{e}$ in order to allow for the correct dissociation limit.

Making all of the assumptions and inferences stated above, the two resulting potential energy functions become:

$$
\begin{aligned}
& V_{\sigma}(r)= \begin{cases}17.77+1.236\left(1-e^{-2.81326 \Delta r}\right)^{2}, & r<r_{e}, \\
17.77+2.319\left(1-e^{-2.05400 \Delta r}\right)^{2}, & r>r_{e},\end{cases} \\
& V_{s}(r)= \begin{cases}18.03+1.236\left(1-e^{-2.81326 \Delta r}\right)^{2}, & r<r_{e}, \\
18.03+2.059\left(1-e^{-2.17983 \Delta r}\right)^{2}, & r>r_{e},\end{cases}
\end{aligned}
$$

where energies are given in $\mathrm{eV}$, distances in $\AA$, and $\Delta r=r-r_{e^{*}}$. These curves resemble that of the $D^{2} \Pi_{g}$ ion for $r<r_{e}$ and switch smoothly at $r=r_{e}$ into curves which dissociate to $\mathrm{N}\left({ }^{4} S_{0}\right)+\mathrm{N}\left({ }^{4} P\right) . \quad V_{\sigma}(r)$ is displayed in Fig. 5 together with potential curves for several other ionic states. $^{33}$

Continuum nuclear wavefunctions for the $V_{\sigma}(r)$ and $V_{r}(r)$ just given were calculated by a Runge-Kutta numerical integration of the corresponding radial Schrödinger equation. The wavefunctions were momentum normalized by requiring the asymptotic form $\sqrt{2 / \pi}$ $\sin (k r+\delta)$, where $\delta$ is a phase shift and $\hbar k$ is the momentum of the nuclear motion asymptotically. ${ }^{57}$ Finally, nuclear wavefunctions for the $\mathrm{N}_{2} X^{1} \Sigma_{g}^{+}$and $\mathrm{N}_{2}^{+} X^{2} \Sigma_{g}^{+}$ bound states were taken to be the Morse oscillator wave-

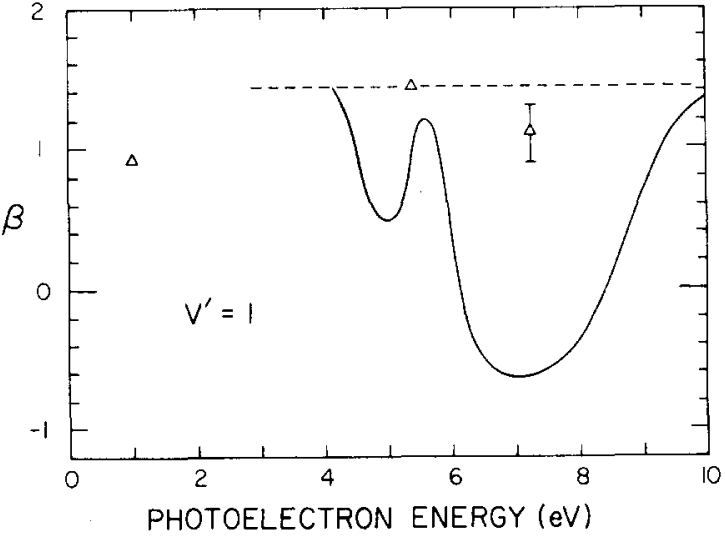

FIG. 8. Variation of $B$ with electron energy for $v^{\prime}=1$ of the $X^{2} \Sigma_{g}^{+}$band as in Fig. 7 .

functions of Nicholls. ${ }^{54}$ These wavefunctions were calculated using spectroscopic parameters from Herzberg. ${ }^{58}$ For the Franck-Condon factors, $F_{\alpha \nu}^{2}$, obtained by squaring the overlap integral between a bound and a momentum-normalized continuum wavefunction, a normalization is expressed as:

$$
\int_{0}^{\infty} F_{\alpha \nu}^{2}(k) d k=1
$$

where $\alpha$ labels the vibrational level of the discrete ionic or neutral nuclear state. Equation (12) allows an independent check on the correctness of the calculation and was satisfied for $\alpha=v^{\prime}=0,1,2$ to within a few percent.

The resulting products of Franck-Condon factors, $F_{i \nu}^{2} F_{\nu v^{\prime}}^{2}$, were calculated as a function of photon energy over a range of 0 to $7 \mathrm{eV}$ above the $20.089 \mathrm{eV}$ dissociation limit, i.e., the 4.51-11.51 eV range of photoelectron energy in the production of $X^{2} \Sigma_{g}^{+}, v^{\prime}=0$. Theoretical results are compared with experimental data points in Figs. 7-9. The model predicts large deviations from $\beta=1.43$ for all vibrational levels, where the minima in $\beta$ occur at different photon energies for different $v^{\prime}$. Furthermore, since for several energy regions, the factors $F_{i \nu}^{2} F_{v v^{\prime}}^{2}$ are similar for $v^{\prime}=0,1,2$, whereas the factors $F_{i v^{\prime}}^{2}$ remain fixed, the model predicts significant

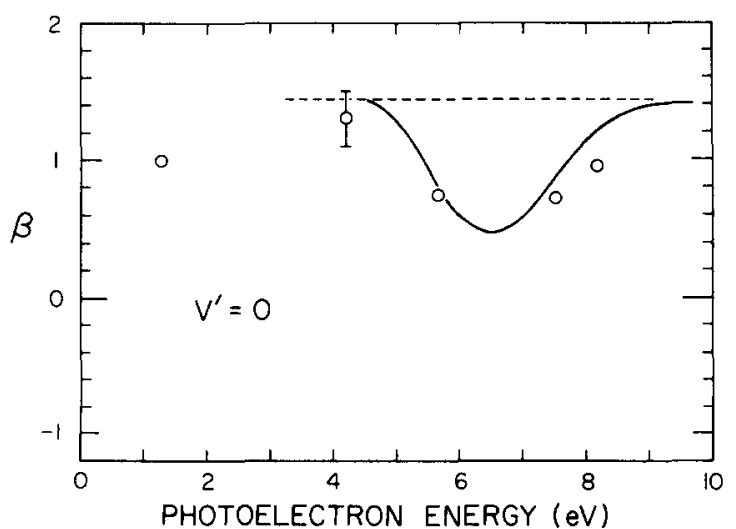

FIG. 7. Variation of $\beta$ with electron energy for $v^{\prime}=0$ of the $X^{2} \Sigma_{g}^{+}$band. Experimental data points are indicated in relation to the calculated variation. The dotted line represents $\beta=1.43$.

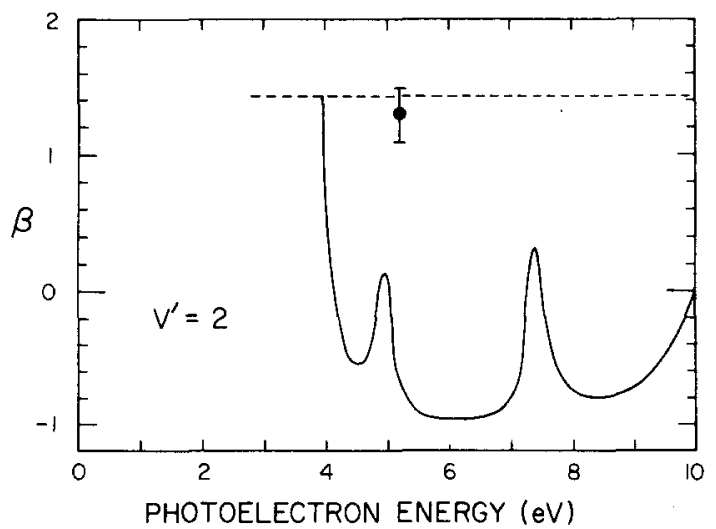

FIG. 9. Variation of $\beta$ with electron energy for $v^{\prime}=2$ of the $X^{2} \Sigma_{g}^{+}$band as in Fig. 7 . 
enhancement of $v^{\prime}=1,2$ for photon energies larger than $21.22 \mathrm{eV}$.

The results of the model calculation for $v^{\prime}=0$ agree reasonably well with experimental results. However, experimental and theoretical results for $v^{\prime}=1$ and 2 do not agree at all. The experimental result for $v^{\prime}=1$ lies siginificantly above that calculated at the electron energy appropriate for $537 \AA$, as seen in Fig。8. The match for $v^{\prime}=2$ peaks at $584 \AA$ in Fig. 9 could probably not be improved by increasing $\beta_{\text {auto }}$ or by shifting the potential curves.

We rationalize the failure of the model to describe accurately the variation of $\beta$ in the following manner. First, we really do not have accurate potential curves for the neutral states in the $20-25 \mathrm{eV}$ region. Recent calculations of Thulstrup and Andersen ${ }^{58}$ and Cartwright and Dunning ${ }^{60}$ have accurately characterized the double excitation ionic states in this region. Rydberg states converging to these ions certainly exist. Second, we only guessed at the theoretical expressions for vibrational intensity given by Eq. (4). Complicated interference cross terms may arise if the exciting radiation is the slightly self-reversed (broadened) output of a rare gas discharge. Finally, autoionization of continuum nuclear states may not be the mechanism for the enhancement of $v^{\prime}=0$ and the different $\beta$ values for $v^{\prime}=0$ and $v^{\prime}=1$.

\section{Other sources for the $X^{2} \Sigma_{g}^{+}$anomaly}

The source of the anomalous behavior of photoelectron intensity in the $v^{\prime}=0$ peak of the $X^{3} \Sigma_{g}^{+}$band and in the variation of the corresponding $\beta$ with photoelectron energy may be due to factors other than autoionization. The electric dipole transition moment may vary with internuclear distance in a way such that its average value is a function of $v^{r}$. Indeed, assuming the validity of the Born-Oppenheimer separation, the transition amplitudes take the following form:

$$
\mathrm{T}_{i f}=\int \chi_{f}(\mathrm{R}) \chi_{i}(R) \mathrm{D}(\mathrm{R}) d^{3} \mathrm{R},
$$

where $R$ is the internuclear vector, $\chi$ are nuclear wavefunctions, and $D(R)$ is the electric dipole transition moment defined by:

$$
\mathrm{D}(\mathrm{R})=\int \psi_{f}(\mathrm{r} ; \mathrm{R}) \mathrm{T}_{\mathrm{op}} \psi_{i}(\mathrm{r} ; \mathrm{R}) d^{3} r
$$

where the $\psi$ are electronic wavefunctions, $\mathbf{T}_{o p}$ is the electric dipole operator, and $\mathbf{r}$ represents the set of electronic orbital and spin coordinates. Frequently, $T$ is factored into a product of a Franck-Condon amplitude and a transition moment by the theorem of the mean:

$$
\mathrm{T}=F_{i f} \overline{\mathrm{D}(\mathrm{R})} \text {. }
$$

The $R$ dependence of $D(R)$ can then yield a dependence of $\overline{D(R)}$ on the vibrational quantum number of the final state.

This effect was investigated previously by Itikawa ${ }^{61}$ and Berkowitz and Spohr ${ }^{62}$ for molecular hydrogen.
Here, relative vibrational intensities in the $X^{2} \Sigma_{g}^{+}$progression of $\mathrm{H}_{2}$ differ significantly from those calculated from accurate Franck-Condon factors. Systematically, over the range of vibrational levels in the ion, the ratio of experimental or accurate (corrected for variations of the electric dipole transition moment with internuclear distance) theoretical vibrational intensities to the Franck-Condon factors increased by $4 \%-5 \%$ with each increasing quantum of vibrational energy in the molecular ion. On the other hand, $\mathrm{Carlson}^{4}$ has reported no change in the asymmetry parameter over the range of vibrational peaks for $\mathrm{H}_{2}$.

The intensity effect observed for $\mathrm{N}_{2}$ is significantly larger than for $\mathrm{H}_{2}$ and the change of $\beta$ is substantial. An explanation for the $\mathrm{N}_{2}$ anomalies based upon changes in the electronic transition moment is nevertheless being suggested by Duzy and Berry。 ${ }^{16}$ We note, however, that the $\beta$ values calculated by Duzy and Berry within $5 \mathrm{eV}$ of ionization threshold disagree markedly with those measured here and previously.

Kalman has recently calculated ${ }^{63}$ the $R$ dependence of $\beta$ for the ${ }^{1} \Sigma_{g}-{ }^{2} \Sigma_{g}^{+}$ionization process in which the $l=1$ outgoing partial wave dominates. Moderate effects are predicted if the difference in equilibrium internuclear separations between the ground and ionic state is small and $0-1$ Franck-Condon factors are likewise small. According to Kalman, the measured difference "in $\beta$ for the $0 \rightarrow 0$ and $0 \rightarrow 1$ peaks of that ionization can be accounted for only in part by the $R$ dependence of the electronic transition moment."

Another possibility is that the shape resonance phenomenon seen in the $K$-shell ionization of $\mathrm{N}_{2}$ may be occurring in the valence shell. Dehmer and Dill ${ }^{64}$ calculate a significant drop in $\beta$ and a corresponding increase in intensity in the region $6-15 \mathrm{eV}$ above ionization threshold for that shell in $\mathrm{N}_{2}$ 。 The $\operatorname{dip}$ in $\beta$ in the region 9-10 eV above threshold is comparable to that observed in the $X^{2} \Sigma_{g}^{+}$band for $v^{\prime}=0$. However, the total intensity enhancement for the $K$-shell appears to be much larger (an increase of $200 \%$ ) than is observed in the $X^{2} \Sigma_{g}^{+}$band. In addition, the magnitude of the effect would have to depend on the vibrational level of the ion if resonance excitation were the mechanism for the present $\mathrm{N}_{2}$ anomaly. Their paper presents no evidence to suggest that the effect could vary with the degree of vibrational level in the ion. Finally, Dehmer and Dill suggest that the effect may be present in core $L$-shell or core $M$-shell ionization. Plummer et al. ${ }^{35}$ associated peaks in the $\mathrm{N}_{2}^{+} X^{2} \Sigma_{g}^{+}$cross section at approximately $28 \mathrm{eV}$ photon energy with a scattering resonance of this type.

\section{D. $B^{2} \Sigma_{u}^{+}$band}

The only $\beta$ values for the $B^{2} \Sigma_{t}^{+}$ionization, measured at $584 \AA$, lie along the curve of the $X^{2} \Sigma_{g}^{+}$ionization. This may be mere coincidence or may indicate that the $\beta$ versus photoelectron energy curves for $X^{2} \Sigma_{g}^{+}$and $B^{2} \Sigma_{u}^{+}$should be the same near ionization threshold, since both ionic states produced are ${ }^{2} \Sigma$ states. 
This possibility is interesting in light of theoretical calculations of photoelectron angular distributions for molecules. Ritchie ${ }^{65}$ has shown that for high photoelectron energies $(>100 \mathrm{eV})$, a torque caused by highorder terms in a multipole expansion about the molecular ionic center has only a small effect. In this energy range, the potential is dominated by a spherically symmetric monopole term and for molecules having a center of symmetry, the form of the angular distribution for ionization from a molecular orbital of gerade and ungerade symmetry is very much different.

Assuming that the wavefunction for the outgoing photoelectron is a plane wave orthogonalized to filled molecular orbitals ${ }^{14}$ yields $\beta$ values for the $B^{2} \Sigma_{u}^{+}$state which are significantly lower than those calculated for the $X^{2} \Sigma_{g}^{+}$state (see Table II). We conclude that no theoretical calculation to date which neglects the role of autoionization via continuum nuclear states can satisfactorily account for the experimental results.

\section{CONCLUSION}

The photoelectron angular distributions have been measured for $\mathrm{N}_{2}$ for photons from both the strong and very weak emission lines of neon and helium glow discharge lamps. The wide range of photon energies covered has allowed us to measure the electron energy dependence of $\beta$.

The variation of $\beta$ with vibrational peaks in the $X^{2} \Sigma_{g}$ photoelectron spectrum at $584 \AA$ is anomalous, as is the variation of $\beta$ with photoelectron energy for the $v^{\prime}=0$ peak of that ionic state. We have discussed several mechanisms for this anomaly. The autoionization pathway through the Rydberg states converging to the $D^{2} \Pi_{g}$ ion might enhance $v^{\prime}=0$ intensity in the $X^{2} \Sigma_{g}^{+}$ionization band by as much as a third and explain the variation of its $\beta$ with energy. However, at present there is no adequate quantitative explanation for the corresponding $\beta$ variation of the $v^{\prime}=1$ and $v^{\prime}=2$ peaks above $20.09 \mathrm{eV}$ threshold for autoionization. An explanation consistent with all experimental results is not available yet. Any mechanism should account for the enhancement of $v^{\prime}=0$ peak intensity relative to the $v^{\prime}=1$ one in the energy region near $21 \mathrm{eV}$, and the anomalous difference between the energy dependences of $\beta$ for the $v^{\prime}=0$ and $v^{\prime}=1$ peaks.

We suggest that further angular distribution experiments at variable wavelength for $\lambda>617 \AA$ corresponding to the $\mathrm{N}\left({ }^{4} S_{0}\right)+\mathrm{N}\left({ }^{4} P\right)$ dissociation limit will help to establish more firmly the trend observed here for the $X^{2} \Sigma_{g}^{+}$ band. In addition, experiments for $\lambda<660 \AA$, corresponding to the threshold for the $\mathrm{N}_{2}^{+} B^{2} \Sigma_{u}^{+}$state, should be performed to examine for further similarities in the energy dependence of $\beta$ for loss of electrons from $\sigma_{g} 2 p$ and $\sigma_{u} 2 s$ orbitals.

\section{ACKNOWLEDGMENTS}

We are grateful to Dr. Thomas $\mathrm{N}_{0}$ Rescigno and Dr. C. William McCurdy for supplying a computer program to calculate momentum-normalized nuclear continuum wavefunctions.
${ }^{1}$ M. I. Al-Joboury and D. W. Turner, J. Chem. Soc. 1863, 5141.

${ }^{2}$ D. W. Turner, C. Baker, A. D. Baker, and C. R. Brundle, Molecular Photoelectron Spectroscopy (Wiley-Interscience, London, 1970).

${ }^{3} \mathrm{~J}$. L. Gardner and J, A. R. Samson, J. Chem. Phys. 60, 3711 (1974).

${ }^{4}$ T. A. Carlson, Chem. Phys. Lett. 8, 23 (1971); T. A. Carlson and A. E. Jonas, J. Chem. Phys. 55, 4913 (1971).

${ }^{5}$ R. Morgenstern, A. Niehaus, and M. W. Ruf in Electronic and Atomic Collisions, edited by L. Branscomb (NorthHolland, Amsterdam, 1971), p. 167.

${ }^{6}$ W. H. Hancock and J. A. R. Samson, J. Electron Spectrose. 9, 211 (1976).

${ }^{7}$ I. G. Kaplan and A. P. Markin, Opt. Spectrosc. 24, 475 (1968); 25, 275 (1968).

${ }^{8}$ M. Borm and J. R. Oppenheimer, Ann. Phys. 84, 457 (1927).

${ }^{9}$ D. C. Cartwright and A. Kuppermann, Phys. Rev. 163, 86 (1967).

${ }^{10} \mathrm{~J}$. W. Cooper and S. T. Manson, Phys. Rev. 177, 157 (1969).

${ }^{11} \mathrm{~J}$. Cooper and R. N. Zare, in Lectures in Theoretical Physics, edited by S. Geltman, K. Mahanthappa, and N. Brittin (Gordon and Breach, New York, 1969), Vol. XI-C, p. 317.

${ }^{12}$ S. T. Manson, J. Electron Spectrose. 1, 431 (1972).

${ }^{13}$ S. Iwata and S. Nagakura, Mol. Phys. 27, 425 (1974).

${ }^{14}$ J. W. Rabalais, T. P. Debies, J. L. Berkosky, J. -T. J. Huang, and F. O. Ellison, J. Chem. Phys. 61, 529 (1974); 62, 4588 (1975).

${ }^{15}$ B. Schneider and R. S. Berry, Phys. Rev. 182, 141 (1969). ${ }^{16}$ C. Duzy and R. S. Berry, J. Chem. Phys. 64, 2421 (1976).

${ }^{17}$ D. Dill, Phys. Rev. A 7, 1976 (1973).

${ }^{18}$ J. A. R. Samson and J. L. Gardner, Phys. Rev. Lett. 31, 1327 (1973).

${ }^{19}$ D. C. Mason, D. M. Mintz, and A. Kuppermann, Rev. Sci. Instrum. 48, 926 (1977).

${ }^{20}$ R. L. Kelley, "Atomic Emission Lines Below 2000 Angstroms, Hydrogen Through Argon," NRL Report No. 6648, Naval Research Laboratory, Washington, D.C., 1968.

${ }^{21}$ L. Åsbrink, Faraday Discuss. Chem. Soc. 64, 142 (1972).

${ }^{22}$ A. W. Potts, T. A. Williams, and W. C. Price, Faraday Discuss. Chem. Soc. 54, 104 (1972).

${ }^{23}$ M. Okuda and N. Jonathan, J. Electron Spectrosc. 3, 19 (1974).

${ }^{24}$ D. M. Mintz and A. Kuppermann, Electronic and Atomic Collisions, edited by J. S. Risley and R. Geballe (University of Washington, Seattle, 1975), p. 567.

${ }^{25} \mathrm{M}$. Nakamura and Y. Iida, Proceedings, IV International Conference on Vacuum-Ultraviolet Radiation Physics, Hamburg, 1974, p. 170.

${ }^{26}$ P. Natalis, J. Delwiche, and J. E. Collin, Chem. Phys. Lett. 13, 491 (1972); J. E. Collin and P. Natalis, Int. J. Spectrom. Ion Phys. 2, 231 (1969).

${ }^{27}$ G. V. Marr and P. R. Woodruff, J. Phys. B 8, L377 (1976).

${ }^{28}$ U. Fano, Phys. Rev. 124, 1866 (1961).

${ }^{29}$ F. H. Mies, Phys. Rev. 175, 164 (1968).

${ }^{30}$ J. N. Bardsley, Chem. Phys. Lett. 2, 329 (1968).

${ }^{31}$ A. L. Smith, Phil. Trans. R. Soc. (London) A 268, 169 (1970); A. L. Smith, J. Quant. Spectrosc. Radiat. Transfer $10,1129(1970)$.

${ }^{32}$ J. A. Kinsinger and J. W. Taylor, Int. J. Mass Spectrom. Ion Phys. 11, 461 (1973).

${ }^{33}$ F. R. Gilmore, J. Quantum Spectrosc. Radiat. Transfer 5, 369 (1965).

${ }^{34}$ J. N. Bardsley, Chem. Phys. Lett. 1, 229 (1967).

${ }^{35} \mathrm{E}$. W. Plummer, T. Gustafson, W. Gudat, and D. E. Eastman, Phys. Rev. 15, 2339 (1977).

${ }^{36} \mathrm{E}$. Lindholm, Ark. Fys. 40, 111 (1968).

${ }^{37} \mathrm{~J}$. Berkowitz and W. A. Chupka, J. Chem. Phys. 51, 2341 (1969).

${ }^{38}$ R. M. White, T. A. Carlson, and D. P. Spears, J. Electron. Spectrose. 3, 59 (1974). 
${ }^{39} \mathrm{D}$. M. Mintz and A. Kuppermann (manuscript in preparation).

${ }^{40} \mathrm{~L}$. C. Lee, R. W. Carlson, D. L. Judge, and M. Ogawa, J. Quant. Spectrosc. Radiat. Transfer 13, 1023 (1973).

${ }^{41}$ M. Sasanuma, E. Ishiguro, Y. Morioka, and M. Nakamura in Conference Digest of the Third International Conference on Vacuum Ultraviolet Radiation Physics, edited by Y. Nakai (Tokyo, Japan, 1971), p. 1 pA 2-3.

${ }^{42}$ W. S. Watson, J. Lang, and D. T. Stewart, J. Phys. B 6, L148 (1973).

${ }^{43} \mathrm{~K}$. Codling, Astrophys. J. 143, 552 (1966).

${ }^{44} \mathrm{~T}$. Namioka, K. Yoshino, and Y. Tanaka, J. Chem. Phys. 38,2629 (1963).

${ }^{45}$ R. W. Nicholls, J. Quant. Spectrosc. Radiat. Transfer 2, 433 (1962).

${ }^{46} \mathrm{~J}$. A. R. Samson and R. B. Cairns, J. Geophys. Res, 69, 4583 (1964).

${ }^{47}$ J. F. M. Aarts and F. J. de Heer, Physica 62, 45 (1971).

${ }^{48}$ M. J. Mumma and E. C. Zipf, J. Chem. Phys. 85, 5582 (1971).

${ }^{49} \mathrm{~J}$. M. Ajello, J. Chem. Phys. 63, 1156 (1970).

${ }^{50}$ W. Sroka, Z. Naturforsch. Teil A 24, 398 (1969).

${ }^{51}$ L. C. Lee, R. W. Carlson, D. L. Judge, and M. Ogawa, J. Chem. Phys. 61, 3261 (1974); Chem. Phys. Lett. 18, 183 (1973).

${ }^{52}$ K. D. Beyer and K. H. Welge, J. Chem. Phys. 51, 5323 (1969).

${ }^{59}$ J. A. R. Samson, G. N. Haddad, and J. L. Gardner, J. Phys. B 10, 1749 (1977).
${ }^{54}$ R. W. Nicholls, J. Res. Natl. Bur. Stand. 65, 451 (1961).

${ }^{55}$ Here we note first that the autoionization of Rydberg states with $\nu=2$ and $\nu=3$ has been implicated by the term value analysis of Sec. V.C. 1. The roughly equal intensity of vibrational peaks for $v^{\prime}=2,3$, and 4 of the $736 \AA$ spectrum in Fig. 3 matches Meinel Franck-Condon factors for neither $v=2$ nor $\nu=3$ alone. Thus, a consideration of some weighted combination of the two autoionizing states may be more valuable. Use of the mean of Meinel band Franck-Condon factors for $v=2$ and $v=3$ yields $Q_{0}^{\text {auto }} / Q_{v^{\prime}}^{\text {anto }}$ in the range $0.5-2$ for $v^{\prime}=2,3$, and 4 .

${ }^{56} \mathrm{C}$. R. Brundle, referred to as a private communication to $T$. A. Carlson in Ref. 4.

${ }^{57} \mathrm{~J}$. R. Taylor, Scattering Theory: The Quantum Theory on Nonrelativistic Collisions (Wiley, New York, 1972), p. 186.

${ }^{58} \mathrm{G}$. Herzberg, Molecular Spectra and Molecular Structure. I. Spectra of Diatomic Molecules (Van Nostrand-Reinhold, New York, 1939).

${ }^{59}$ E. W. Thulstrup and A. Andersen, J. Phys. B 8, 965 (1975).

${ }^{60}$ D. C. Cartwright and T. H. Dunning, Jr., J. Phys. B 8, L100 (1975).

${ }^{61}$ Y. Itikawa, J. Electron. Spectrosc. 2, 125 (1973).

${ }^{62} \mathrm{~J}$. Berkowitz and R. Spohr, J. Electron Spectrosc. 2, 143 (1973).

${ }^{68}$ O. F. Kalman, Mol. Phys. 34, 397 (1977).

${ }^{64} \mathrm{~J}$. L. Dehmer and D. Dill, Phys. Rev. Lett. 36, 213 (1975).

${ }^{65}$ B. Ritchie, J. Chem. Phys. 61, 3291 (1974). 